\title{
SISTEMAS DE PARENTESCO DE LA RAMA HUASTECANA
}

\author{
Rosa Elena Anzaldo Figueroa \\ Dirección de Lingüística, INAH
}

El presente trabajo expone los resultados de una investigación que tiene varios objetivos. El primero de ellos es hacer un estudio descriptivo de los sistemas terminológicos de parentesco correspondientes a la rama huastecana de la familia mayense. Esta rama comprende los dialectos potosino y veracruzano de la lengua huaxteca o teenek, así como el chicomucelteco o cabil, lengua de los habitantes de Chicomucelo, Comalapa, Yayagüita y Huitatán, Chiapas (Ruz, 1989: 12-15) y que se habló hasta la década de los veinte del siglo pasado. Un segundo objetivo es la clasificación de los sistemas descritos y el objetivo final es efectuar un análisis comparativo de las terminologías y de las estructuras de los sistemas parentales.

Los estudios sobre la rama huastecana son numerosos. Sucintamente mencionaré algunas referencias: Kaufman (1972: 13), en su estudio glotocronológico, informa que hace 4200 años se inició la fragmentación del protomaya y que fue la rama huastecana la primera que se separó. Esto ocurrió antes del Clásico, ya que este grupo no comparte con el resto de la familia los rasgos culturales característicos de ese periodo (Suárez, 1995: 229). Sobre las propuestas relacionadas con el desplazamiento de los pueblos mayas están, por una parte, la que afirma que se efectuó un movimiento paulatino de los grupos mayas, de norte a sur, y los huastecos o teenek permanecieron en el norte (Swadesh, 1960: 236 y Manrique, 1989: 211-212) y, por la otra, la que considera que Los Altos de Guatemala fueron el lugar de origen de los mayas, y que desde ahí los huastecos emigraron hacia el norte (Kroeber, 1944: 160).

Como se observa, no hay consenso sobre si los huastecos o teenek permanecieron en el norte (supuesto lugar de origen) o si se desplazaron hacia el norte desde Los Altos de Guatemala. Tampoco lo hay, consecuentemente, en lo relacionado con el movimiento del grupo chicomucelteco o cabil. La separación glotocronológica del huasteco y el chicomucelteco da la fecha plausible de 1100 de nuestra era y se presume que los chicomuceltecos emigraron hacia el sur (Suárez, 1995: 228); Swadesh (1960: 235-236) fecha la divergencia interna de la rama huastecana -en huasteco o teenek y chicomucelteco o cabil- no antes de los últimos 10 o 15 siglos. Kroeber (op.cit., p.161) quien como vimos considera el sur como el lugar original de los mayas, dice que no es clara la interpretación histórica, porque: o el grupo chicomucelteco fue un fragmento huasteco rezagado cuando viajaron hacia el noroeste desde el territorio principal de los mayas, o ambos grupos se desprendieron del cuerpo principal maya y, mientras los huaste- 
cos continuaron su desplazamiento, los chicomuceltecos, después de un tiempo, retornaron al sur. Y para abundar, Manrique (op.cit.: 211-212) comenta que durante la penetración huasteca hacia el sur, una parte de este grupo - supuestamente los chicomuceltecos- no pudo asentarse en las tierras nuevas, porque éstas ya estaban ocupadas, por lo que continuaron su viaje hasta ubicarse en la ribera meridional del Grijalva.

Cabe señalar que este desprendimiento hacia el sur se inscribe en un periodo durante el cual se efectuaron otras migraciones semejantes, como la de los pipiles en $800 \mathrm{~d}$. C., la del grupo chiapaneco-mangue poco antes del $900 \mathrm{~d}$. C. y la de los tlapanecos y subtiabas en 1100 d.C. (Suárez, 1995: 226-228). Campbell (1979: 908) coincide, en general, con los fechamientos de Suárez, pero calcula que para los pipiles el movimiento ocurrió en 900 d.C.

Pasando ahora al material objeto de estudio, se dispone de la información procedente de dos documentos que proporcionan los primeros datos que se tienen sobre el huasteco. El primero es la Doctrina Christiana en la lengua Guasteca (1571) del fraile agustino Juan de la Cruz, personaje del cual se sabe solamente que predicó durante largos años en la Huasteca y que fue en el convento de Huejutla, Hidalgo, donde concluyó la doctrina en cuestión. Tampoco se conocen su lugar de origen, la fecha de su llegada a la Nueva España, o el lugar y el año de su fallecimiento (Ochoa, 1995: 122). Por tratarse de un escrito religioso de poca extensión se encontraron en él apenas nueve términos de parentesco.

El segundo documento es el Paradigma Apologético y Noticia de la Lengua Huasteca con Vocabulario, Catecismo y Administración de Sacramentos (1985), obra debida a Tapia Zenteno, de quien se supone nació antes de 1690 en la ciudad de México y se estima murió después de 1767. Fue alumno del Seminario Tridentino Metropolitano y bachiller en filosofia y teología; realizó una labor amplia como párroco, vicario, juez, protonotario, comisario y catedrático de lengua mexicana en la Real y Pontificia Universidad, y dominaba a la perfección las lenguas huasteca y nahua. El Paradigma se refiere al dialecto potosino, aunque no se sabe con exactitud la procedencia geográfica del material lingüístico. Este documento proporcionó 49 términos de parentesco y se le puede considerar representativo de toda la región huasteca potosina, lugar donde el autor permaneció largo tiempo, y que comprende los municipios de Tampamolón, Villa de los Valles y San Antonio, este último fundado por el mismo Tapia Zenteno (Montejano y Aguiñaga, 1985: 50-84).

Los datos de Siloxúchil, municipio de Tantoyuca, Veracruz, los obtuvo Guiteras (1948: 152-172), quien nos proporciona 34 términos de parentesco. Con respecto a las terminologías más recientes, éstas son producto del trabajo de campo que realicé en 1995 en la comunidad de Tamaletón, municipio de Tancanhuitz, San Luis Potosí, el cual permitió obtener un total de 50 términos; y en Loma Larga, comunidad perteneciente al municipio de Tantoyuca, Veracruz, que proporcionó 44 términos. Posteriormente, en 1997 realicé una visita a la comunidad de San Francisco, del municipio de Chontla, Veracruz, considerada por algunos investigadores como lugar de hablantes de una tercera variante del huasteco (comunicación personal de Roberto Zavala), logrando elicitar 33 términos de parentesco. Para la obtención de las terminologías se empleó el método genealógico y listas complementarias. Los datos demográficos se tomaron del Censo General de Población y Vivienda (INEGI, 1990).

Sobre el chicomucelteco contamos con el material que obtuvieron Sapper en 1894 (1954: 295-320) y Termer en 1926 (1930: 926-936), agregando los datos que proporciona Zimmermann (1966: 27-71) posteriormente, con lo que se tienen 17 términos de parentesco. 
La investigación se inicia con la descripción del material, identificando las piezas léxicas de cada sistema con una clave consistente en un número y una letra. El número distingue los términos del sistema y la letra designa la variante en cuestión. Asimismo, a cada terminología se le asigna un nombre. Lo anterior queda expuesto en el Cuadro siguiente:

Cuadro 1. Identificacion de los sistemas de parentesco de la rama huastecana

\begin{tabular}{|l|l|c|}
\hline Procedencia de los datos & Nombre del sistema & Clave del sistema \\
\hline Juan de la Cruz & Siglo xvı & A \\
\hline Tapia Zenteno & Siglo xviII & B \\
\hline Chicomucelo & Chicomucelteco & C \\
\hline Siloxúchil, Veracruz & Siloxúchil & D \\
\hline San Luis Potosí & Tamaletón & E \\
\hline Veracruz & Loma Larga & F \\
\hline Chontla, Veracruz & San Francisco & G \\
\hline
\end{tabular}

Los términos de parentesco contemporáneos son referenciales. En cuanto a los términos de los siglos xvl, xvII y del chicomucelteco, considerando que sus autores no lo especifican, se los ha estimado referenciales dada su vigencia como tales en los sistemas actuales.

La transcripción de los términos de Siloxúchil, de Tapia Zenteno, del cabil o chicomucelteco y del siglo xvı se presentan respetando la ortografía de las fuentes documentales; las demás terminologías se representan también ortográficamente para hacer más transparente la comparación. ${ }^{1}$ Es común que en las lenguas mesoamericanas los términos de

' Es conveniente revisar aquí las dos propuestas que se han hecho para las grafias de los fonemas de la lengua huasteca, porque apoyan las correspondencias que se hacen entre las grafias de los fonemas del huasteco y las que usan los otros autores en las páginas que anteceden los cuadros 6,7,8 y 9 . Una de las propuestas es para la variante de Veracruz (Ochoa, 1984: 11-31) que considera 21 consonantes más seis préstamos del español ( $\mathrm{d} g$ f s r r r y 10 vocales.

Consonantes

\begin{tabular}{|c|c|c|c|c|c|}
\hline p & $t$ & C & $\check{c}$ & k & $\mathrm{k}^{\mathrm{w}}$ \\
\hline & $\mathrm{t}^{\prime}$ & $c^{\prime}$ & $\check{c}^{\prime}$ & $k^{\prime}$ & $k^{k}$ \\
\hline b & (d) & & & (g) & \\
\hline (f) & $\theta$ & (s) & ร̌ & & \\
\hline $\mathrm{m}$ & $\mathrm{n}$ & & & & \\
\hline w & & 1 & $y$ & & \\
\hline & & $(\mathrm{r}, \check{\mathrm{f}})$ & & & \\
\hline
\end{tabular}

Vocales

i, i: u, u:

e, e: 0,0 :

a , a:

ANZALDo Figueroa / SISTEMAS DE PARENTESCO DE LA RAMA HUASTECANA

Estudios de Cultura Maya. Vol. XXIII, 2003

Instituto de Investigaciones Filológicas/

Centro de Estudios Mayas, UNAM

ISSN 0185-2574 
parentesco se expresen poseídos; sin embargo, en este trabajo, con objeto de igualar el material reciente con el que se obtuvo de los autores mencionados, se omite el proclítico pronominal posesivo.

Una vez terminada la descripción de los siete sistemas de parentesco se procederá al análisis y clasificación de los mismos. Aquí cabe señalar que la clasificación del sistema del siglo xvı no se realiza, porque la insuficiencia de los datos no permite su estructuración. Por lo que se refiere al chicomucelteco, aunque también se cuenta con pocos elementos, se clasificará el sistema con los datos disponibles.

Este estudio parte de la propuesta que hizo Kroeber (1952: 175-181), ${ }^{2}$ quien consideró necesario encontrar los principios o categorías que subyacen a las relaciones de parentesco.

El análisis componencial de Goodenough (1956: 195-216) derivado de las categorías de Kroeber y el estructural formal de Lounsbury (1964a:193-211 y 1964b: 212-255), ${ }^{3}$

La otra variante es la potosina, en la que Edmonson (1988: 30-31) propone 26 consonantes, incluidos tres préstamos del español ( $\mathrm{d} \mathrm{g}$ f) y 10 vocales.

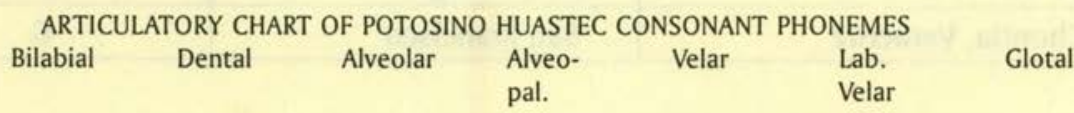

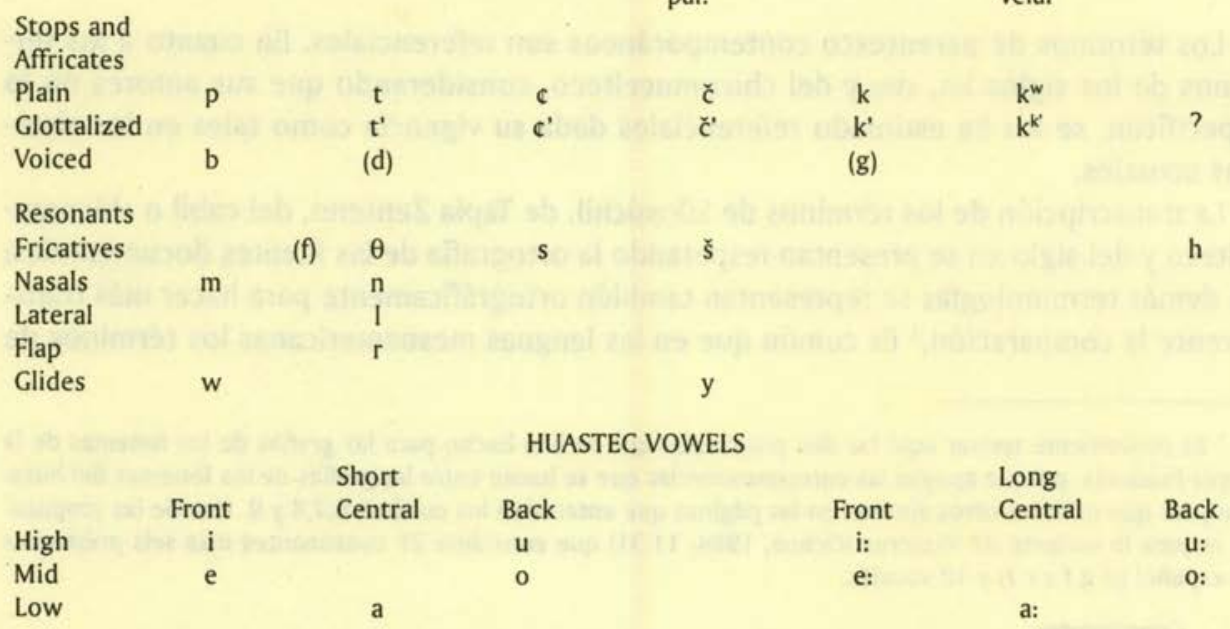

${ }^{2}$ Kroeber propone ocho categorías que subyacen a los términos de parentesco: generación, consanguíneo/afin, lineal/colateral, sexo del hablante, sexo del pariente, edad relativa dentro de una misma generación, sexo de la persona que da la relación de parentesco y condición de vida de la persona que relaciona.

${ }^{3}$ El análisis componencial de Goodenough, inspirado en las categorías de Kroeber, descompone los términos en un conjunto de rasgos pertinentes que funcionan como variables, cuyos valores verifica en cada uno de los términos, de forma que la intersección de los valores definirá el significado de los lexemas. La propuesta de Lounsbury consiste en ocho términos de parentesco primarios: P 'padre', M 'madre', Ho 'hermano', Ha 'hermana', ho 'hijo', ha 'hija', Eo 'esposo', Ea 'esposa' y dos designadores sexuales (masculino ?, femenino ?). La síntesis de dos significados primarios genera términos secundarios. Por ejemplo: en español, los significados primarios Ha ('hermana') y Eo ('esposo') nos dan dos términos secundarios: HaEo (hermana del esposo,'cuñada') y EoHa (esposo de la hermana, 'cuñado'). La síntesis de tres significados primarios origina términos terciarios: ha ('hija),Ho ('hermano') y M ('madre') (hija del hermano de la madre,'prima'). Los designadores indican el sexo del hablante cuando preceden a los términos y cuando en los diagramas aparecen después del número y clave que identifican el término, están indicando el sexo del pariente. 
permitirán realizar el análisis semántico de los términos y así determinar su significado. Para establecer los tipos de los sistemas de parentesco de las lenguas en estudio se utilizarán tanto la propuesta de Lowie (1946: 84-89) como la de Murdock (1965: 223). ${ }^{4}$ Asimismo se compararán las piezas léxicas tanto en su forma como en su significado. El objeto de esta comparación es identificar los términos cognados, las posibles innovaciones y señalar los desplazamientos. Con respecto a estos últimos se tomará en cuenta el criterio cronológico, por lo que primero se considerará como punto de partida los nueve términos del 'Siglo xvi' y después la terminología de 'Tapia Zenteno'. Los tipos de desplazamiento son: a) sustitución o reemplazo de un referente por otro, b) extensión (generalización) y c) reducción de referentes (Jeffers y Lehiste, 1982: 126-137). Todo lo anterior permitirá observar las relaciones que existen entre los sistemas de parentesco de la rama huastecana y, con ello, obtener las conclusiones pertinentes.

\section{Doctrina christiana en la lengua Guasteca (1571) de fray Juan de la Cruz}

Fue en el convento de Huejutla donde quedó concluida la Doctrina en septiembre de 1570. La impresión se hizo en la casa de Pedro Ocharte un año después, 1571. Dado que se trata de un documento religioso poco extenso, el número de términos de parentesco que se pudo extraer para incorporarlos en el presente estudio se limitó a nueve. Antes de proceder a su examen es conveniente hacer notar que los denotados $\mathrm{P}$, ho y Eo aparecen repetidos. Es decir, para designar al padre está documentado tanto el término paylom, como pap; para el hijo, atic y tam; para el esposo, illau y pizob. Al respecto cabe subrayar que, en el siglo xvil, Tapia Zenteno reportó los términos paylom y atic para el hablante masculino, pap y tam para el femenino y pizob para el caso del cónyuge. Aun y cuando en la Doctrina no se especifiquen estas distinciones, no es aventurado suponer que sí eran pertinentes en el siglo xvi, por lo cual se ha tomado la decisión de incorporarlas en el análisis.

\section{Términos primarios: 8}

Puros (3)

1A. M mim "madre", 2A.Ho quiçab "hermano", 3A.Eo(a) pizob "esposo/ a" o "cónyuge". ${ }^{5}$

Designador sexual (6)

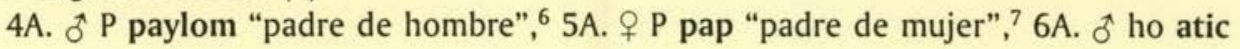

\footnotetext{
${ }^{4}$ Lowie propone cuatro tipos de acuerdo con los términos que se dan a los parientes masculinos de la primera generación (padre, tío paterno y tío materno): generacional, fusional bifurcado, colateral bifurcado y lineal. Por su parte Murdock lo hace con seis tipos dependiendo de los términos que nombran a los parientes femeninos de la generación cero (hermana, prima paralela y prima cruzada): hawaiano, sudanés, esquimal, iroqués, omaha y crow, éstos dos últimos son variantes del iroqués.

5 Tapia Zenteno (1985: 165) lo reporta para 'cónyuge'.

${ }^{6} \mathrm{lbid}$. para el hablante masculino.

${ }^{7}$ Ibid. para el hablante femenino.
} 
"hijo de hombre", 7A. + ho tam "hijo de mujer", 8A. ô Eo illau "esposo", 9A. + Ea vxmal "esposa".

\section{Los nueve términos antes descritos son elementales. ${ }^{8}$}

A continuación, para definir el significado de los lexemas de acuerdo con el análisis componencial de Goodenough, se considerarán los siguientes rasgos: generación, línea directa, afinidad, sexo del pariente y sexo del hablante. En el diagrama 1 quedan representados esos rasgos.

Diagrama 1. Análisis componencial de la terminología de parentesco obtenida por fray Juan de la Cruz

\begin{tabular}{|c|c|c|c|c|c|}
\hline \multirow[t]{2}{*}{ G } & \multirow[t]{2}{*}{$\begin{array}{l}E \\
G \\
O\end{array}$} & \multicolumn{2}{|c|}{$\begin{array}{l}\text { Línea } \\
\text { Directa }\end{array}$} & \multicolumn{2}{|c|}{ Cónyuge } \\
\hline & & 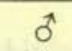 & 우 & $\sigma^{*}$ & ㅇ \\
\hline+1 & $\begin{array}{l}0 \\
q\end{array}$ & $\begin{array}{l}4 \mathrm{~A} \\
5 \mathrm{~A}\end{array}$ & $1 \mathrm{~A}$ & & \\
\hline 0 & $\begin{array}{l}0 \\
q\end{array}$ & $2 A$ & & $8 \mathrm{~A}$ & $9 \mathrm{~A}$ \\
\hline-1 & $\sigma^{\pi}$ & $6 \mathrm{~A}$ & & & \\
\hline
\end{tabular}

Dado el escaso material del que se dispone, solamente se puede advertir que el sistema cuenta con tres generaciones: ${ }^{9}$ la de ego $\mathrm{G}^{\circ}$, una ascendente $\mathrm{G}^{1}$ y una descendente $\mathrm{G}^{-1}$.

Considerando que faltan los términos que permiten clasificar el sistema de acuerdo con las tipologías de Lowie (hermano del padre y hermano de la madre) y de Murdock (hermana, prima cruzada y prima paralela), ${ }^{10}$ aquí queda concluido el análisis de este sistema.

\footnotetext{
${ }^{8}$ Para Murdock (1965:98) un término elemental es una palabra irreductible que no puede ser analizada en componentes de elementos léxicos con significados parentales

${ }^{9}$ La categoría generación se representa con la letra G y con un superíndice numérico se indica a qué nivel corresponde.

${ }^{10} \mathrm{Pr} / /$, primos paralelos, son los hijos del hermano del padre y los hijos de la hermana de la madre, en tanto que PrX, primos cruzados, son los hijos del hermano de la madre y los hijos de la hermana del padre.
} 


\section{Sistema de parentesco potosino de la lengua Huasteca, según Tapia Zenteno (Siglo xviI)}

Como se apuntó antes, la información sobre los términos de parentesco del huasteco potosino se obtuvo de la obra de Carlos de Tapia Zenteno, Paradigma Apologético y Noticia de la Lengua Huasteca con Vocabulario, Catecismo y Administración de Sacramentos, editado por René Acuña en 1985. A continuación se listan los términos de parentesco que proceden del diccionario, de la Administración de Sacramentos que acompaña a la Noticia, o de su fuente directa en el facsímil. La consulta de este último fue de gran utilidad para dilucidar algunos puntos que no estaban claros en la transcripción moderna.

De la obra se seleccionaron 49 términos, los cuales describen con mucha precisión el sistema de parentesco. Sin embargo, es necesario aclarar algunos aspectos del significado exacto de algunos de ellos.

a) El autor indica que el término $22 \mathrm{~B}$ itzan se refiere al esposo de la tía y al tío, sin especificar si este último es tío paterno o materno. Actualmente en los dialectos de 'Tamaletón' y de Veracruz (exceptuando 'San Francisco' donde designa tanto al tío paterno como al materno) este término se refiere al tío materno, por lo cual se estima pertinente que también en este sistema designe a ese pariente ya que, por otra parte, Tapia Zenteno sí proporciona términos para el hermano del padre que expresan las categorías sexo del hablante y edad relativa, aplicando al hermano mayor los términos $34 \mathrm{~B} \delta^{\star}$ yelam paylom y $35 \mathrm{~B}$ + yelam pap y al hermano menor los términos $36 \mathrm{~B} \delta$ to tzutz paylom y $37 \mathrm{~B}+$ tzutz pap.

b) En el texto consultado hay un solo término para la tía (23B tzanub), y el autor tampoco aclara de cuál se trata, si de la tía materna o de la paterna. En los sistemas contemporáneos antes citados - igual que en el caso del tío paterno- existe un término especial para la hermana de la madre mas no para la del padre, que se nombra de la misma manera que el resto de las tías, y es justamente el término 23B tzanub. Pero a diferencia de lo que sucede con el tío, donde se tienen cinco términos que permiten diferenciar al tío materno del paterno, para las tías proporciona solamente un término, por lo que se procederá a destinarlo tanto a la rama materna como a la paterna (como sucede en 'San Francisco').

c) Tapia Zenteno reporta que los hijos de los tíos se consideran como hermanos. Es decir, ego masculino nombra al primo con los mismos términos que da al hermano mayor (15B $\delta^{\star}$ at mim) y al hermano menor (12B $\delta^{\hat{\alpha}}$ atatal), según se trate del hijo mayor o menor de los tíos; y a la prima con el término de la hermana (7B + ixam). En el caso de ego femenino, el autor comenta que comúnmente se emplea para los primos el término $47 \mathrm{~B}$ q epchial y $48 \mathrm{~B}$ q ebal.

d) No se obtuvo término alguno para la hija ni para la sobrina y, en cuanto al sobrino, proporciona los términos que corresponden a los parientes cruzados, es decir, con 25B itzac iyac ego masculino designa al hijo de la hermana y ego femenino se refiere al hijo del hermano. Al respecto cabe agregar que Guiteras (1948: 152-172), en su estudio sobre el sistema de parentesco huasteco de Siloxúchil, reporta que ambos egos denotan a los sobrinos paralelos con el término de los hijos (chakámel) y que los sobrinos cruzados reciben otro término (ichák), que resulta ser el mismo que da el documento (25B itzac), 
habida cuenta del empleo que esta variante hace de la ch (africada, alveopalatal, sorda) en los términos que Tapia Zenteno transcribe con la ts (africada, alveolar, sorda). Es probable que la ausencia de términos para los sobrinos paralelos se deba a que los consideraban como hijos, aunque este detalle es dudoso que pasara inadvertido a tan concienzudo investigador. Actualmente en Tamaletón los dos egos nombran a todos los sobrinos con el término 20E itsak' que neutraliza la categoría sexo del pariente y que es también el término ya citado de Tapia Zenteno. No obstante, en el análisis me ceñí puntualmente a lo descrito por el autor.

e) Se considera que el término que designa a los bisnietos (44B tzabilvmomob), parientes de la generación $\mathrm{G}^{-3}$, es un término genérico atendiendo a que, con algunas excepciones para los términos de los hijos, universalmente el resto de los parientes de las generaciones descendentes neutralizan la categoría sexo del pariente.

Una vez hechas las aclaraciones pertinentes, se procede a la descripción de los 49 términos del sistema:

Términos primarios (19)

Puros (3)

1B.M mim "madre", 2B.Eo(a) tomol "esposo/a", 3B.Eo(a) pizob "esposo/a"

\section{Designador sexual (16)}

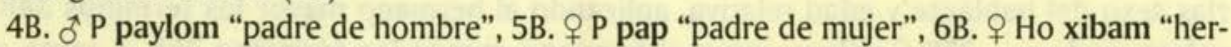
mano de mujer", 7B. $\delta^{*} \mathrm{Ha}, \delta^{\hat{\alpha}}$ haHo(a)P(M) ixam "hermana y prima, de hombre", 8B. $\delta^{*}$ ho atic "hijo de hombre", 9B. + ho taam "hijo de mujer", 10B. + Eo illauh "esposo", 11B. ơ Ea ixal "esposa", 12B. ô $\mathrm{Ho}<$, hoHo(a)P(M) atatal "hermano menor y primo, de hombre"," 13B. $+\mathrm{Ha}>$ bayil "hermana mayor de mujer", 14B. $+\mathrm{Ha}<\mathrm{acab}$ "hermana menor de mujer"

Derivativos $^{12}$

15B. $\sigma^{\top} \mathrm{Ho}>$, hoHo(a)P(M) at mim "hermano mayor y primo, de hombre", 16B. $\delta^{\wedge}$ ho> cochim atic "hijo mayor de hombre", 17B. ô ho< tzutzu atic "hijo menor de hombre", 18B. ㅇ ho > cochim tam "hijo mayor de mujer", 19B. ㅇ ho< tzutz tam "hijo menor de mujer"

Términos secundarios (21)

Puros (5)

20B. PP(M) mam "abuelo", 21B. MP(M) ach "abuela", 22B. HoM itzan "tío materno", 23B. HaP(M) tzanub "tía"

Derivativo

24B. EaP mimle "madrastra" menor.

" La categoría edad relativa dentro de una misma generación se marca con los símbolos > mayor y <

${ }^{12}$ Términos derivativos son los compuestos de un término de parentesco y de otro elemento que no es de parentesco (Murdock, 1965: 98). Por ejemplo: 19B. $q$ ho< tzutz tam 'hijo menor de mujer', es tzutz "pequeño" y tam "hijo". 


\section{Designador sexual (16)}

25B. $\sigma^{*}$ hoHa, $q$ hoHo itzac iyac "sobrino, hijo de la hermana dice el hombre; sobrino, hijo del hermano, dice la mujer", 26B. ơ ho(a) ho(a) momob "nieto/a de hombre", 27B. 우 ho(a)ho(a) iyib,"nieto/a de mujer", 28B. ô PEa,Eoha iyam "suegro de hombre, yerno", 29B. + PEo,Eaho alib "suegro de mujer, nuera", 30B. $\delta^{\top}$ EoHa, $\delta^{\top} \mathrm{HoEa}$ bay "cuñado de hombre", 31B. + EaHo, o HaEo xahuan "cuñada de mujer"

\section{Derivativos}

32B. $\sigma^{\top}$ MEa uxum iyam "suegra de hombre", 33B. + MEo uxum alib "suegra de mujer", 34B. $\sigma^{\hat{~}} \mathrm{Ho}>\mathrm{P}$ yelam paylom "tío paterno mayor dice el hombre", 35B. $+\mathrm{Ho}>\mathrm{P}$ yelam pap "tío paterno mayor dice la mujer", 36B. $\sigma^{\wedge} \mathrm{Ho}<\mathrm{P}$ tzutz paylom "tío paterno menor dice el hombre", 37B. $\&$ Ho <P tzutz pap "tío paterno menor dice la mujer", 38B. ô EoM paylomle "padrastro de hombre", 39B. + EoM paple "padrastro de mujer", 40B. † EoHa,HoEo, ơ EaHo,HaEa at mul "cuñado de mujer, cuñada de hombre".

\section{Términos terciarios (9)}

Puros (6)

Derivativos

41B. PEo(a)ho(a) yetzel atsii "consuegro", 42B. PP(M)P(M) tzabilmam "bisabuelo", 43B. MP(M)P(M) tzabilach "bisabuela", 44B. ho(a)ho(a) ho(a) tzabilvmomob "bisnieto/ a", 45B. EoHaEo(a) atiyam "concuño", 46B. MEo(a)ho(a) uxum atzii "consuegra"

\section{Designador sexual (3)}

47B. + ho(a)Ho(a)P(M) epchial "primo/a de mujer", 48B. $q$ ho(a) Ho(a)P(M) ebal "primo/a de mujer"

\section{Derivativo}

49B. ㅇ EaHoEo at alib "concuña de mujer"

Del ordenamiento anterior se deduce que 15 términos son derivativos y 34 elementales.

Siguiendo a Lounsbury, a partir de los términos primarios se derivaron los secundarios y los terciarios lineales, ${ }^{13}$ que es el límite hasta el que llega el sistema de parentesco huasteco de Tapia Zenteno. Los rasgos que permiten obtener el significado de los lexemas son los siguientes: generación, línea directa, colateral paterno, colateral materno, afinidad (por los cónyuges, por parte de los parientes del cónyuge, por los cónyuges de los parientes, por los parientes de los cónyuges de los parientes y por los cónyuges de los parientes de los cónyuges), el sexo del hablante y el sexo del pariente. La interrelación de todos estos rasgos se ve en el diagrama 2 , el cual permite tener una visión completa del sistema.

\footnotetext{
${ }^{13}$ Los términos colaterales, por ejemplo, hohoHoP, hohohoHoP, etcétera, no se consideran términos cuaternarios, quinarios, etcétera.
} 
Diagrama 2. Análisis componencial de la terminología de parentesco del huasteco obtenida por Tapia Zenteno

\begin{tabular}{|c|c|c|c|c|c|c|c|c|c|c|c|c|c|c|c|c|c|}
\hline \multirow[t]{2}{*}{ G } & \multirow[t]{2}{*}{$\begin{array}{l}E \\
G \\
0\end{array}$} & \multicolumn{2}{|c|}{$\begin{array}{l}\text { Línea } \\
\text { Directa }\end{array}$} & \multicolumn{2}{|c|}{$\begin{array}{l}\text { Colateral } \\
\text { Paterno }\end{array}$} & \multicolumn{2}{|c|}{$\begin{array}{l}\text { Colateral } \\
\text { Materno }\end{array}$} & \multicolumn{2}{|c|}{$\begin{array}{c}\text { Cónyuge } \\
\text { del } \\
\text { Pariente }\end{array}$} & \multicolumn{2}{|c|}{$\begin{array}{c}\text { Pariente } \\
\text { del } \\
\text { Cónyuge }\end{array}$} & \multicolumn{2}{|c|}{ Cónyuge } & \multicolumn{2}{|c|}{$\begin{array}{l}\text { Pariente } \\
\text { Cónyuge } \\
\text { Pariente }\end{array}$} & \multicolumn{2}{|c|}{$\begin{array}{l}\text { Cónyuge } \\
\text { Pariente } \\
\text { Cónyuge }\end{array}$} \\
\hline & & 0 & 우 & 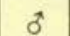 & 우 & $0^{*}$ & † & $\sigma^{\circ}$ & 우 & $\sigma^{\circ}$ & 우 & $0^{\circ}$ & 우 & $\sigma^{0}$ & 우 & $\sigma^{*}$ & q \\
\hline+3 & $\begin{array}{l}\text { o } \\
+\end{array}$ & $42 B$ & $43 B$ & & & & & & & & & & & & & & \\
\hline+2 & के & $20 B$ & $21 B$ & & & & & & & 47 & 0. & & & & & & \\
\hline+1 & $\sigma^{2}$ & $4 B$ & 1 & $\begin{array}{l}34 \mathrm{~B}> \\
36 \mathrm{~B}< \\
\\
35 \mathrm{~B}> \\
37 \mathrm{~B}<\end{array}$ & $23 B$ & $22 \mathrm{~B}$ & 23B & 39B & $24 B$ & 28B & $32 \mathrm{~B}$ & & & 7 & & & r \\
\hline 0 & (1) & $\begin{array}{c}12 B< \\
15 B> \\
6 B\end{array}$ & $\begin{array}{c}7 B \\
13 B> \\
14 B<\end{array}$ & -1 & $\begin{array}{l}\text { B } 0.1 \\
-47 \mathrm{E}\end{array}$ & $48 \mathrm{~B}-$ & \&- & $\overline{5}$ & $\mathrm{~B} \delta$. & OB $q-$ & $\overline{1}$ & $\begin{array}{c}-2 \mathrm{~B} \\
10 \mathrm{~B}\end{array}$ & $\begin{array}{l}11 \mathrm{~B} \\
\mathrm{~B}-\end{array}$ & $41 B$ & 46B & 45B & $y$ \\
\hline-1 & 更 & $\begin{array}{c}8 \mathrm{~B} \\
16 \mathrm{~B}> \\
17 \mathrm{~B}< \\
\\
9 \mathrm{~B} \\
18 \mathrm{~B}> \\
19 \mathrm{~B}<\end{array}$ & & $25 \mathrm{~B}$ & 25B & & & $28 \mathrm{~B}$ & $29 B$ & & & 27 & & & & & 4 \\
\hline-2 & क & $\begin{array}{r}-26 \\
-27 \\
\end{array}$ & $\begin{array}{r}B- \\
- \\
\end{array}$ & . & & & & & . & & & & & & & & \\
\hline-3 & $\sigma^{\circ}$ & -44 & $B$ & $t^{2}$ & & $=$ & & 14 & wat & & & & & & & & \\
\hline
\end{tabular}

Este sistema presenta las siguientes características importantes:

a. Consta de siete generaciones.

b. Hay terminología específica para cada ego, pues la categoría sexo del hablante se expresa en un alto número de términos que alcanza un $71 \%$ del total de vocablos.

c. Uso extensivo de la categoría edad relativa dentro de una misma generación.

d. Términos recíprocos entre suegro y yerno (28B iyam), y suegro y nuera (29B alib). 
A continuación, con fundamento en las tipologías de Lowie y de Murdock se clasifica el sistema. De acuerdo con el primero, el cual se basa en los términos masculinos de la primera generación ascendente: padre, tío paterno y tío materno, el sistema se clasifica como de tipo colateral bifurcado, por designar a cada pariente con un término diferente, como se muestra a continuación:

$$
\begin{aligned}
& \text { Ego ô: : P 4B paylom }+{ }^{14} \\
& \text { HoP Ho }>\text { P 34B yelam paylom, Ho }<\text { P } 36 \text { B tzutz paylom }+ \\
& \text { HoM 22B itzan } \\
& \text { Ego } q \text { : } \mathrm{P} 5 \mathrm{~B} \text { pap + } \\
& \text { HoP Ho }>\text { P 35B yelam pap, Ho<P 37B tzutz pap }+ \\
& \text { HoM 22B itzan }
\end{aligned}
$$

Y en lo que se refiere a Murdock, el sistema es tipo hawaiano para ego masculino, ya que un término nombra a las tres parientes, que son la hermana, la prima paralela y la prima cruzada:

\section{Ego $\delta^{\text {t }}:$ Ha $7 \mathrm{~B}$ ixam $=$ \\ $\mathrm{Pr} / / 7 \mathrm{~B}$ ixam $=$ \\ $\operatorname{PrX} 7 \mathrm{~B}$ ixam}

En cambio, para ego femenino el sistema es tipo esquimal porque ambas primas se designan con el mismo término, que es diferente del de la hermana:

$$
\begin{aligned}
& \text { Ego } \text { \& : Ha Ha }>13 \mathrm{~B} \text { bayil, } \mathrm{Ha}<14 \mathrm{~B} \text { acab }+ \\
& \operatorname{Pr} / / 47 \mathrm{~B} \text { epchial } \sim 48 \mathrm{~B} \text { ebal }= \\
& \operatorname{PrX} 47 \mathrm{~B} \text { epchial } \sim 48 \mathrm{~B} \text { ebal }
\end{aligned}
$$

\section{Sistema de parentesco del chicomucelteco o cabil}

Se comentó antes que el material objeto de estudio es el que obtuvieron, en una primera instancia, Sapper en 1894 (1954: 295-320), ${ }^{15}$ que comprende 347 palabras (de las cuales 17 son términos de parentesco) y Termer en 1926 (1930: 926-936), ${ }^{16}$ que proporciona 287 vocablos, 13 de ellos de parentesco. Posteriormente Zimmermann (1955: 27 71) incrementó este material a través de dos medios: uno fue el Manuscrito Sapper de la Biblioteca Latinoamericana de Berlín que proporcionó 161 palabras, de las cuales 75 eran nuevas. El otro consistió en las notas de campo de Termer, que éste cedió a Zimmermann y que aumentó el material en una docena de palabras. Del total de ellas solamente dos son nuevos términos de parentesco y corresponden a los datos de Sapper.

\footnotetext{
${ }^{14}$ Símbolo que indica 'no es igual'.

${ }^{15}$ Agradezco a Michael Knapp King el apoyo que me brindó con la traducción de este artículo.

${ }^{16}$ También a Emmie Hoebens le expreso mi gratitud por el mismo motivo.
} 
A continuación se presenta una lista comparando los 17 lexemas de Sapper, los 13 de Termer y los dos de Sapper que localizó Zimmermann, con el objeto de hacer más evidentes las identidades, las similitudes o las diferencias entre los términos de parentesco:

\begin{tabular}{|c|c|c|c|}
\hline $\begin{array}{l}\text { Términos de } \\
\text { parentesco }\end{array}$ & Sapper & Termer & Zimmermann \\
\hline $\begin{array}{l}\text { esposo } \\
\text { esposa } \\
\text { padre } \\
\text { madre } \\
\text { hijo } \\
\text { hija } \\
\text { hermano> } \\
\text { hermano< } \\
\text { abuelo } \\
\text { abuela } \\
\text { suegro } \\
\text { suegra } \\
\text { cuñado } \\
\text { cuñada } \\
\text { tío } \\
\text { tía } \\
\text { nieto, a } \\
\text { papá } \\
\text { yerno } \\
\text { hermana } \\
\text { hermano } \\
\text { primo, a }\end{array}$ & $\begin{array}{l}\text { tetlom } \\
\text { uxmal } \\
\text { ajtic calé } \\
\text { epé } \\
\text { tamtic utam } \\
\text { tam } \\
\text { cocú ucucú } \\
\text { acaú } \\
\text { mam } \\
\text { xepin xipin } \\
\text { iyam } \\
\text { iyam } \\
\text { úai } \\
\text { atmún } \\
\text { ichau } \\
\text { xapin } \\
\text { iú }\end{array}$ & $\begin{array}{l}\text { uxmál } \\
\text { palé (español) } \\
\text { epé (del aguacateco II) }{ }^{17} \\
\text { vatík } \\
\text { atíc } \\
\text { vakám } \\
\text { uxapín } \\
\text { uyám } \\
\text { ichán } \\
\text { ajtic } \\
\text { uyám } \\
\text { nunú } \\
\text { ucú }\end{array}$ & 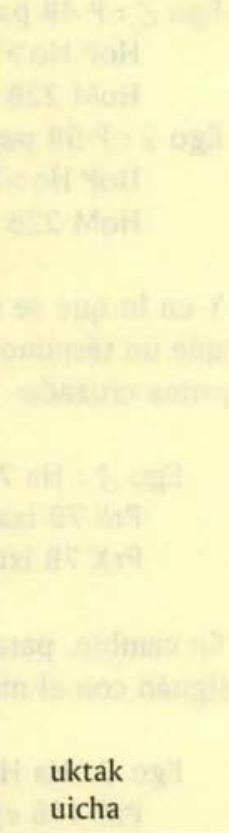 \\
\hline
\end{tabular}

Sapper (1954: 295-320) comenta que entrevistó a dos hablantes procedentes de diferentes localidades, pero no especificó su sexo. Termer (1930: 926-936), por su parte, informa que trabajó con dos personas del sexo masculino. Puesto que se ignora si alguna de las personas entrevistadas fue del sexo femenino, entonces no se puede analizar la categoría sexo del hablante en los términos documentados.

Con objeto de resolver esta situación, es importante tener en cuenta que, en general, no todos los términos expresan la categoría sexo del hablante. De hecho la mayoría de ellos son empleados por ambos sexos. Sin embargo, teniendo en cuenta que para los hijos Sapper da el término tamtic utam $\sim$ tam y Termer el término vatík atíc, y que se ha visto en el sistema 'Siglo xv' que los términos 6A. ồ ho atic "hijo de hombre" y 7A. + ho tam "hijo de mujer", y en el 'Tapia Zenteno' los términos $8 \mathrm{~B}$. $\widehat{o}$ ' ho atic "hijo de hombre" y 9B. + ho taam "hijo de mujer", sí expresan la categoría sexo del hablante, es probable que Sapper haya obtenido para los hijos el término que emplea el hablan-

\footnotetext{
${ }^{17}$ Nadie después de Otto Stoll ha encontrado algo similar al Aguacateco II que, en realidad, fue el invento de una informante que se desempeñaba como su ayudante en las labores domésticas (Campbell 1979: 953).
} 
te de sexo femenino. En consecuencia se estima pertinente atribuir el término de Sapper al hablante femenino y el término de Termer al masculino. Por otra parte, se le asigna a ego masculino aquel término que claramente le corresponde (uxmal 'esposa'); igualmente se le asigna al ego femenino el término correspondiente (itetlom 'esposo') y a ambos sexos los restantes, dado que ni Sapper ni Termer indican lo contrario. De esta manera el análisis se ciñe estrictamente a la descripción de dichos autores. No obstante, es conveniente señalar que en las otras lenguas de la rama huastecana es relevante la categoría sexo del hablante.

Se seleccionan 16 términos de parentesco de la lista de Sapper, porque reporta el mayor número de ellos, cinco de Termer y los dos términos de Zimmermann, sumando en total 23 términos. Incorporando el término de la hija al del hijo, tanto en Sapper como en Termer, el de la suegra al del suegro y el de la tía al de la abuela en la terminología se Sapper, ya que se trata en cada caso del mismo lexema, la lista de 23 términos se reduce a 19:

\begin{tabular}{|c|}
\hline \multirow[t]{2}{*}{ Sapper } \\
\hline \\
\hline 2. padre …....................................jtic calé \\
\hline 3. madre .......................................é \\
\hline 4. suegro, a .....................................iyam \\
\hline 5. hijo, a ......................................mtíc $\sim$ utam $\sim$ tam \\
\hline 6. cuñado .....................................ui ú \\
\hline 7. cuñada .......................................tmún \\
\hline 8. hermano .................................. cocú $\sim$ ucucú \\
\hline 9. hermano < .................................... acaú \\
\hline 10. esposa ........................................uxmal \\
\hline 11. tía, abuela \\
\hline 12. abuelo ......................................... mam \\
\hline 13. nieto, a .................................... iú \\
\hline
\end{tabular}

Y agregando los dos términos de Sapper que obtuvo Zimmermann,
14. hermano uktak
15. primo, a uicha

\section{Termer}
16. hijo, a
atíc
17. yerno
uyám
18. hermana nunú
19. tío ichán

Considerando que Termer da para el yerno el mismo término que Sapper obtuvo para los suegros, entonces quedan finalmente 18 lexemas que constituyen la base para la descripción. Por otra parte, se hace necesario mencionar algunas precisiones relacionadas con los siguientes términos: 
ichán.- En los dialectos de 'Tamaletón', 'Siloxúchil y 'Loma Larga', este término designa al hermano de la madre. Tapia Zenteno no especifica claramente el denotado, sin embargo al observar la totalidad del sistema, cabe suponer que este término corresponde a ese pariente y así se estimó. En consecuencia no sería improbable que el chicomucelteco también lo asignara específicamente al hermano de la madre. ${ }^{18}$

xepin $\sim$ xipin $\sim$ xapin.- tiene dos referentes: la tía y la abuela. El único elemento para asignarlo a determinado pariente es el hecho de que ambos autores coinciden al informar que corresponde a un pariente lineal de la $\mathrm{G}^{2}$, lo que descarta a la tía, pariente colateral de la $\mathrm{G}^{1}$. Si bien Termer obtuvo este término para el abuelo, Sapper, por su parte, proporciona para este mismo pariente el término mam, el cual está vigente en 'Tamaletón'. Esto hace probable que también designara al abuelo en el chicomucelteco. Así las cosas, se podría, con las debidas reservas, asignar a la abuela el término xepin.

De acuerdo con los criterios expuestos antes, se hace la descripción de la terminología donde se ha seleccionado un término cuando hay alternantes:

\section{Términos primarios (10)}

Puros (6)

1C. P ajtic "padre", 2C. M epé "madre", ${ }^{19}$ 3C. Ho uktak "hermano", 4C. Ha nunú "hermana", 5C. Ho> cocú "hermano mayor", 6C. Ho< acaú "hermano menor"20

Designador sexual (4)

7C. ô ho(a) atíc, 8C. ㅇ ho(a) tam "hijo/a", 9C. ㅇ Eo tetlom "esposo", 10C. ỡ Ea uxmal "esposa"

\section{Términos secundarios (7)}

Puros (7)

11C.PP(M) mam "abuelo", 12C. MP(M) xepin "abuela", 13C. HoM ichau "tío materno", 14C. ho(a)ho(a) iú "nieto/a”, 15C. EoHa,HoEo(a) úai “cuñado", 16C. P(M)Eo(a), Eoha iyam "suegro/a, yerno"

\section{Derivativo}

17C. EaHo,HaEo(a) atmún "cuñada"21

${ }^{18}$ Los datos disponibles dejan vacío 'hermano del padre'. Al respecto pudo haber sucedido que el término específico no haya sido registrado por ninguno de los investigadores, o bien que 13C.ichán también designara al tío paterno, tal como sucede en la actualidad en San Francisco, Chontla. El término ichan en otras lenguas tiene distintos referentes. Por ejemplo, en el ch'ol (Esponda, 1996: 105-120) designa al hermano menor tanto del padre como de la madre y además al hijo del hermano de la madre. En el maya-yucateco de los siglos xvı (Borodatova y Kozhanovskaya, 1999: 332-364) y xx (Anzaldo y Briceño, 1998: 203-219), en el maya mopán (Schumann, 1997: 36) y en el pokomam del siglo xvı (Miles, 1983: 81-82) ese término se refiere al esposo, aunque en esta última lengua también nombra al hermano del esposo. En el cakchiquel (Blair, 1981: 480-481) denomina al cuñado, hermano del esposo y esposo de la hermana de ego femenino. Un estudio más amplio de este tema, que no es posible tratar ahora, podrá aportar mayor información sobre la función de este término en el sistema de parentesco de las lenguas mayas.

${ }^{19}$ Este término no se encuentra en el resto de la rama huastecana, por lo que es probable que haya sido difundido del tojolabal, que para designar a la madre usaba el vocablo xep (Ruz, 1982: 153).

${ }^{20}$ Zimmermann (1955:61) inciso $2 . u \Rightarrow b$, acab, bai.

${ }^{21}$ Zimmermann (op. cit: :65) inciso 5.n P I, atmul. 
Términos terciarios (1)

Puros (1)

18C. ho(a)Ho(a)P(M) uicha "primo/a"22

Aplicando la clasificación que hace Murdock de los términos de parentesco, 17 términos son elementales y uno derivativo.

Pasando al análisis componencial se determinan los siguientes rasgos: generación, línea directa, colateral paterno, colateral materno, afinidad por los cónyuges, por los cónyuges de los parientes y por los parientes de los cónyuges. En el diagrama 3 queda representado lo anterior.

Diagrama 3. Análisis componencial de la terminología de parentesco del chicomucelteco o cabil obtenida por Sapper y Termer.

\begin{tabular}{|c|c|c|c|c|c|c|c|c|c|c|c|c|c|}
\hline \multirow[t]{2}{*}{ G } & \multirow[t]{2}{*}{$\begin{array}{l}\text { E } \\
\text { G } \\
\text { O }\end{array}$} & \multicolumn{2}{|c|}{$\begin{array}{l}\text { Línea } \\
\text { Directa }\end{array}$} & \multicolumn{2}{|c|}{$\begin{array}{l}\text { Colateral } \\
\text { Paterno }\end{array}$} & \multicolumn{2}{|c|}{$\begin{array}{l}\text { Colateral } \\
\text { Materno }\end{array}$} & \multicolumn{2}{|c|}{$\begin{array}{c}\text { Cónyuge } \\
\text { del } \\
\text { Pariente }\end{array}$} & \multicolumn{2}{|c|}{$\begin{array}{c}\text { Pariente } \\
\text { del } \\
\text { Cónyuge }\end{array}$} & \multicolumn{2}{|c|}{ Cónyuge } \\
\hline & & 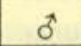 & 우 & $\delta$ & 우 & $\delta$ & 우 & $\sigma$ & 우 & 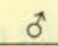 & 우 & $\sigma^{\circ}$ & 우 \\
\hline+2 & के & $10 \mathrm{C}$ & $11 \mathrm{C}$ & & & & & & & & & & \\
\hline+1 & $\begin{array}{l}0 \\
+\end{array}$ & $1 \mathrm{C}$ & $2 \mathrm{C}$ & & & & $12 \mathrm{C}$ & & & - & & 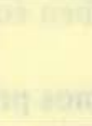 & \\
\hline 0 & 우 & $\begin{array}{l}3 C \\
6 C \\
7 C\end{array}$ & $4 C$ & $\longrightarrow$ & -1 & 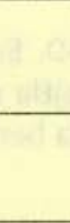 & $\bar{U}$ & (17) & & 160 & & $8 \mathrm{C}$ & 9C \\
\hline-1 & $\begin{array}{l}0 \\
+\end{array}$ & -5 & $C-$ & & & & & $15 \mathrm{C}$ & & & & & \\
\hline-2 & $\begin{array}{l}0 \\
q\end{array}$ & -13 & $C-$ & & & & & & & & & & 17 \\
\hline
\end{tabular}

El diagrama permite destacar los aspectos notables de este sistema que son:

a. Dado que la terminología es limitada, el sistema solamente tiene la generación de ego $\left(\mathrm{G}^{\circ}\right)$, dos ascendentes $\left(\mathrm{G}^{1}, \mathrm{G}^{2}\right)$ y dos descendentes $\left(\mathrm{G}^{-1}, \mathrm{G}^{-2}\right)$, es decir, cinco generaciones.

\footnotetext{
22 Este término designa al genérico primos, pero en los otros sistemas se refiere al genérico sobrinos, en
} Veracruz ichak' y 'Tamaletón' itsak'; en 'Tapia Zenteno' itzac y en 'Siloxúchil' ichák para los sobrinos cruzados. 
b. El término que designa a la madre (2C epé) es un préstamo del tojolabal (cf. nota 19). Actualmente esa lengua designa a la madre con el préstamo nan (Ruz, 1982: 153).

c. Hay un término recíproco (16C iyam) que se refiere a los suegros y al yerno, con la peculiaridad de que nombra al suegro y a la suegra, cuando en los demás sistemas (excepto el del 'Siglo xvi' que no lo proporciona) hay un lexema para cada uno de ellos.

d. Se marca la edad relativa dentro de una misma generación en los términos que designan al hermano (5C cocú 'hermano mayor', 6C acaú 'hermano menor').

En cuanto a la clasificación del sistema, no se puede aplicar la tipología de Lowie porque falta el término del tío paterno. De acuerdo con Murdock, el sistema es esquimal, como se muestra a continuación:

Ha $4 \mathrm{C}$ nunú $\neq$

$\mathrm{Pr} / / 18 \mathrm{C}$ uicha $=$

PrX 18C uicha

IV. Sistema de parentesco del huasteco de Siloxúchil, Veracruz

Este material fue obtenido por la antropóloga Guiteras (1948: 152-172) durante una estancia de 45 días en el municipio de Tantoyuca, Veracruz. Procede de la congregación de Siloxúchil, que es una de las 27 del citado municipio. El huasteco que se habla en Veracruz es otro dialecto de la lengua huasteca. Este sistema consta de 34 términos que se describen en seguida:

\section{Términos primarios (9)}

Puros (5)

1D. P taata "padre", 2D. M naana "madre", 3D. Eo(a) tomkíl "esposo/a", 4D. Ho(a) kizáb "hermano/a", 5D. ho(a), ở ho(a)Ho, ๆ ho(a)Ha chakámel "hijo/a, sobrinos, hijos del hermano dice el hombre; sobrinos, hijos de la hermana, dice la mujer"

Designador sexual (4)

6D. ô Ho, ơ hoHo(a)P(M) atál "hermano y primo, de hombre", 7D. 우 Ho, 우 hoHo(a)P(M) chu'ch "hermano y primo, de mujer, 8D. ơ Ha, ơ ha Ho(a)P(M) ixám "hermana y prima, de hombre, 9D. ㅇ Ha, 우 haHo(a)P(M) bayil "hermana y prima, de mujer"

\section{Términos secundarios (20)}

Puros (12)

10D. HoP paylóm "tío paterno", 11D. HaM mim "tía materna", 12D. HaP chanúb "tía paterna",13D. HoM ichán "tío materno",14D. ho(a) ho(a),ho(a)ho(a) ho(a) mómob "nieto/a, bisnieto/a", 15D. Eoha iyám "yerno", 16D. Eaho alíb "nuera"

\section{Derivativos}

17D. PP(M) pulék-taata "abuelo", 18D. MP(M) pulék-naana "abuela", 19D. EoM chab taata "padrastro", 20D. EaP chab naana "madrastra", 21D. ho(a)Eo(a) chab chakámel "hijastro/a" 
Designador sexual (8)

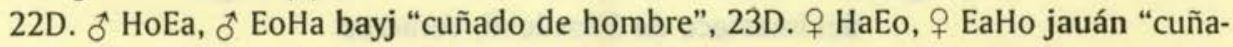
da de mujer", 24D. $\delta^{*}$ ho(a)Ha, + ho(a)Ho ichák "sobrinos, hijos de la hermana, dice el hombre; sobrinos, hijos del hermano, dice la mujer"

Descriptivos $^{23}$

25D. ô PEa taata-iyám "suegro de hombre", 26D. ํ PEo taata-alíb "suegro de mujer", 27D. $\delta^{-1}$ MEa naana-iyám "suegra de hombre", 28D. + MEo naana-alíb "suegra de mujer"

\section{Derivativo}

29D. ồ HaEa, ô EaHo, + HoEo, ㅇ EoHa at-mul "cuñada de hombre, cuñado de mujer"

Términos terciarios (5)

Puros (3)

Derivativos

30D. $P P(M) P(M)$ chab pulék-taata "bisabuelo", 31D. MP(M)P(M) chab pulék-naana "bisabuela", 32D. PEo(a)ho(a) at-chij "consuegro"

Designador sexual (2)

Derivativos

33D. ơ EoHaEa at-iyám "concuño de hombre", 34D. ๆ EaHoEo at-alíb "concuña de mujer"

En este sistema se encontraron 19 términos elementales, 11 derivativos y cuatro descriptivos, distribuidos todos ellos en siete generaciones.

Los rasgos pertinentes para aplicar el análisis componencial son: generación, línea directa, colateral paterno, colateral materno, afinidad (por los cónyuges, por los parientes cónyuge, por los cónyuges de los parientes, por los parientes de los cónyuges de los parientes y por los cónyuges de los parientes de los cónyuges), el sexo del hablante y el sexo del pariente. Todos estos rasgos quedan plasmados en el diagrama 4.

${ }^{23}$ Términos descriptivos son los compuestos por dos términos de parentesco (Murdock 1965: 98). Por ejemplo 28D. ㅇ MEo naana-alíb "suegra de mujer", naana "madre", alíb "nuera". 
Diagrama 4. Análisis componencial de la terminología del huasteco de Siloxúchil, Veracruz

\begin{tabular}{|c|c|c|c|c|c|c|c|c|c|c|c|c|c|c|c|c|c|}
\hline \multirow[t]{2}{*}{ G } & \multirow[t]{2}{*}{$\begin{array}{l}\text { E } \\
\text { G } \\
\text { O }\end{array}$} & \multicolumn{2}{|c|}{$\begin{array}{l}\text { Línea } \\
\text { Directa }\end{array}$} & \multicolumn{2}{|c|}{$\begin{array}{l}\text { Colateral } \\
\text { Paterno }\end{array}$} & \multicolumn{2}{|c|}{$\begin{array}{l}\text { Colateral } \\
\text { Materno }\end{array}$} & \multicolumn{2}{|c|}{$\begin{array}{c}\text { Cónyuge } \\
\text { del } \\
\text { Pariente }\end{array}$} & \multicolumn{2}{|c|}{$\begin{array}{c}\text { Pariente } \\
\text { del } \\
\text { Cónyuge }\end{array}$} & \multicolumn{2}{|c|}{ Cónyuge } & \multicolumn{2}{|c|}{$\begin{array}{l}\text { Pariente } \\
\text { Cónyuge } \\
\text { Pariente }\end{array}$} & \multicolumn{2}{|c|}{$\begin{array}{l}\text { Cónyuge } \\
\text { Pariente } \\
\text { Cónyuge }\end{array}$} \\
\hline & & 0 & 우 & $0^{7}$ & 우 & $\sigma^{\circ}$ & q & 0 & 웅 & 0 & q & $0^{*}$ & f & 0 & q & 0 & 운 \\
\hline+3 & क & 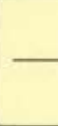 & -30 & $\mathrm{D}$ 6. 31 & 1D $9-$ & 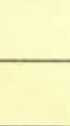 & & & & & & & & & & & \\
\hline+2 & ๙ & 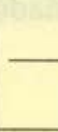 & -17 & D o .1 & 18D 우 & & & 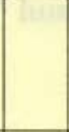 & 34 & & t. & & & & & & \\
\hline+1 & o & 1D & 2D & $10 \mathrm{D}$ & $12 \mathrm{D}$ & 13D & 11D & 19D & 20D & $\begin{array}{l}25 \mathrm{D} \\
26 \mathrm{D}\end{array}$ & $\begin{array}{l}27 D \\
28 D\end{array}$ & & & & & & \\
\hline 0 & $\begin{array}{l}0 \\
0\end{array}$ & $\begin{array}{r}6 \mathrm{D} \\
4 \\
7 D^{4}\end{array}$ & & - & $\begin{array}{l}\text { 6D oे. } \\
\text { 7D के. }\end{array}$ & & & - & $\begin{array}{l}22 \mathrm{D} \text { क } \\
\text { 290 के }\end{array}$ & $\begin{array}{l}, 29 D \\
23 D\end{array}$ & $\begin{array}{l}q- \\
q-\end{array}$ & & & 32D & & 33D & $34 \mathrm{D}$ \\
\hline-1 & $\begin{array}{l}0 \\
+\end{array}$ & $5 \mathrm{D}$ & & $\longrightarrow$ & $-5 D, 2$ & 24D - & $\overline{-}$ & $15 \mathrm{D}$ & 16D & -2 & $1 \mathrm{D}$ & 69 & & $9 x$ & & & 80 \\
\hline-2 & के & & & & & & & & & & & & & 2 & & & \\
\hline-3 & के & & & & & & & & & & & & & & & & \\
\hline
\end{tabular}

La observación del diagrama permite hacer los siguientes comentarios:

a. Los términos se distribuyen en siete generaciones.

b. Hay un término genérico (4D kizáb) para los hermanos y además términos específicos que expresan la categoría sexo del hablante, para hermano (6D $\sigma^{\star}$ atál, 7D 우 chu'ch) y hermana (8D $\delta$ ixám, 9D + bayil), términos que se extienden a los primos, sean paralelos o cruzados.

c. Es el único sistema que diferencia a los sobrinos paralelos - que se designan como a los hijos- (5D chakámel) y a los sobrinos cruzados (24D ichák).

d. Para los cuñados están 22D ơ bayj 'cuñado'y 23D + jauán 'cuñada' con los que se refieren al pariente de su mismo sexo y con otro, 29D at-mul, ambos egos nombran al pariente de sexo opuesto. En los dialectos de 'Tapia Zenteno', 'Tamaletón' y 'San Francisco' existen cuatro lexemas para cuñados. 
Procediendo a la clasificación del sistema, de acuerdo con la tipología de Lowie, es colateral bifurcado:

P $1 D$ taata $\neq$

HoP 10D paylóm $\neq$

HoM 13D ichán

Si se sigue la tipología de Murdock, entonces el sistema es hawaiano:

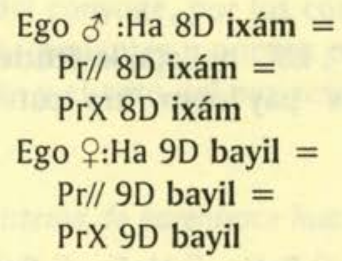

Guiteras (1948: 163) estima que es un sistema de parentesco de tipo clasificatorio o fusional bifurcado (Lowie, 1946: 84-89) que ha sufrido modificaciones por accidentes históricos y aculturación.

V. Sistema de parentesco del huasteco de Tamaletón, San Luis Potosí

Tancanhuitz de Santos es uno de los 56 municipios del Estado de San Luis Potosí; cuenta con 18483 habitantes, de los cuales 6152 hablan huasteco. En el presente trabajo se estudia el sistema de parentesco del dialecto que se habla en la comunidad de Tamaletón, perteneciente al municipio antes citado.

A continuación se hace la descripción del material obtenido en el campo, que consta de 50 términos:

\section{Términos primarios (15)}

Puros (10)

1E. P taata "padre", 2E. P pay'loom "padre", 3E. M naana "madre", 4E. M miim "madre", 5E. Ha,haHo(a)P(M) ebchal "hermana, prima", 6E. ho kwitoolil "hijo", 7E. ha ts'ik'aachil "hija", 8E. ho(a) atik "hijo/a", 9E. ho(a) tsakaamil "hijo/a", 10E. Eo(a) tomkiil "esposo/a"

\section{Designador sexual (5)}

11E. $\delta$ Ho, ơ hoHo(a)P(M) ataatal "hermano y primo, de hombre", 12E. $q$ Eo ilow "esposo", 13E. $\widehat{o}$ Ea iixal "esposa" 


\section{Derivativo}

14E. $\delta$ Ho at miim "hermano de hombre", ${ }^{24} 15 \mathrm{E}$. $q \mathrm{Ho}$, + hoHo(a)P(M) inik ebchal "hermano y primo, de mujer"

\section{Términos secundarios (21)}

Puros (13)

16E.PP(M) maam "abuelo", 17E.MP(M) aach "abuela", 18E.HoM itsaan "tío materno", 19E. HaP tsanuub "tía paterna", 20E. ho(a) Ho(a) itsak' "sobrino/a", 21E. ho(a)ho(a) momob "nieto/a", 22E. Eaho al'iib "nuera"

\section{Derivativos}

23E.EoM tatle' "padrastro", 24E.EaP mimle' "madrastra", 25E. hoEo(a) kwitotle' "hijastro", 26E.haEo(a) ts'ik'achle"'hijastra", 27E.HoP ts'uts' pay'loom "tío paterno", 28E.HaM ts'uts' miim "tía materna"

Designador sexual (8)

29E. $\delta \hat{~ E o H a, ~} \widehat{o}$ HoEa baay "cuñado de hombre", 30E. + EoHa, $q$ HoEo, $q$ EoHaEo xibaam "cuñado y concuño, de mujer", 31E. ơ EaHo, ơ HaEa, ôे EaHoEa ixaam "cuñada y concuña, de hombre" 32E. $\uparrow$ EaHo, $q$ HaEo jawan "cuñada de mujer", 33E. $\delta$ PEa,Eoha iyaam "suegro de hombre, yerno"

\section{Derivativos}

34E. + PEo inik al'iib "suegro de mujer", 35E. $\delta^{+}$MEa uxum iyaam "suegra de hombre", 36E. $q$ MEo uxum al'iib "suegra de mujer"

\section{Términos terciarios (11)}

Puros (6)

Derivativos

37E.haEo(a)P(M) ebchale' "hermanastra", 38E.PP(M)P(M) tsabil maam "bisabuelo", 39E.MP(M)P(M) tsabil aach "bisabuela", 40E. ho(a) ho(a)ho(a) tsabil momob "bisnieto"

\section{Descriptivos}

41E. PEo(a)ho(a) kumpaale atsii "consuegro", 42E. MEo(a)ho(a) kumaale atsii "consuegra"

\section{Designador sexual (5)}

43E. ㅇ EaHoEo bayil "concuña de mujer"

\section{Derivativo}

44E. $\sigma^{\hat{~}}$ hoEo(a)P(M) atatle' "hermanastro de hombre", 45E. $\sigma^{\dagger}$ EoHaEa at iya "concuño de hombre", 46E. $\delta^{\star}$ EoHaEa at muul "concuño de hombre", 47E. + hoEo(a)P(M) inik ebchale' "hermanastro de mujer"

\footnotetext{
${ }^{24}$ Con este término se refiere ego masculino al medio hermano materno.
} 
Términos cuaternarios (3)

Puros (3)

Derivativos

48E. $\mathrm{PP}(\mathrm{M}) \mathrm{P}(\mathrm{M}) \mathrm{P}(\mathrm{M})$ oxil maam "tatarabuelo", 49E. MP(M)P(M)P(M) oxil aach "tatarabuela", 50E. ho(a)ho(a)ho(a)ho(a) oxil momob "tataranieto/a"

Del listado anterior se desprende que 30 términos son elementales, 18 derivativos y dos descriptivos (con préstamos del parentesco ritual español).

El análisis componencial se hace con base en los siguientes rasgos: generación, línea directa, colateral paterno, colateral materno, afinidad (por los cónyuges, por los parientes del cónyuge, por los cónyuges de los parientes, por los parientes de los cónyuges de los parientes y por los cónyuges de los parientes de los cónyuges), el sexo del hablante y el sexo del pariente. Véase diagrama 5 .

\section{Sistema de parentesco huasteco de Loma Larga, Veracruz}

Tantoyuca es uno de los 207 municipios del estado de Veracruz y cuenta con 415 localidades. La población total del municipio es de 85277 habitantes, de los cuales 35902 hablan la lengua huasteca. Loma Larga es una de sus comunidades y se localiza, aproximadamente, $15 \mathrm{~km}$ al noroeste de la cabecera municipal. A continuación se describen los 44 términos de parentesco que se obtuvieron en el campo:

\section{Términos primarios (9)}

Puros (8)

1F.P taata "padre", 2F.M naana "madre", 3F.ho kwitoolil "hijo", 4F.ha tsidhaanil "hija”, 5F.ho(a) chakaamil "hijo/a", 6F.Eo(a) tomtal "esposo/a", 7F.Eo(a) tomkiil "esposo/ a", 8F.Ha, + Ho kidhaab "hermana, hermano de mujer"

Designador sexual (1)

9F. $\delta^{\star} \mathrm{Ho}, \delta^{\star}$ hoHo(a)P(M), $\delta^{\star}$ EoHaEa ataal "hermano, primo y concuño, de hombre"

\section{Términos secundarios (21)}

Puros (14)

10F. HoM ichaan "tío materno", 11F. HaP chanuub "tía paterna", 12F HaM,MEo(a) miim "tía materna, suegra", 13F. ho(a)Ho(a) ichak' "sobrino/a", 14F. ho(a)ho(a) momob "nieto/a", 15F. Eoha iyaam "yerno", 16F. Eaho al'iib "nuera"

\section{Derivativos}

17F.PP(M) pulek taata "abuelo", 18F.MP(M) pulek naana "abuela", 19F. EoM chab taata "padrastro", 20F. EaP chab naana "madrastra", 21F. hoEo(a) chab kwitoolil "hijastro", 22F. haEo(a) chab tsidhaanil "hijastra"

\section{Descriptivo}

23F. EaP miim naana "madrastra" 


\section{Diagrama 5. Análisis componencial de la terminología del huasteco de Tamaletón, San Luis Potosí}

\begin{tabular}{|c|c|c|c|c|c|c|c|c|c|c|c|c|c|c|c|c|c|}
\hline \multirow[t]{2}{*}{ G } & \multirow[t]{2}{*}{$\begin{array}{l}\text { E } \\
G \\
\text { O }\end{array}$} & \multicolumn{4}{|c|}{$\begin{array}{l}\text { Línea Colateral } \\
\text { Directa Paterno }\end{array}$} & \multicolumn{2}{|c|}{$\begin{array}{l}\text { Colateral } \\
\text { Materno }\end{array}$} & \multicolumn{2}{|c|}{$\begin{array}{c}\text { Cónyuge } \\
\text { del } \\
\text { Pariente }\end{array}$} & \multicolumn{2}{|c|}{$\begin{array}{c}\text { Pariente } \\
\text { del } \\
\text { Cónyuge }\end{array}$} & \multicolumn{2}{|c|}{ Cónyuge } & \multicolumn{2}{|c|}{$\begin{array}{l}\text { Pariente } \\
\text { Cónyuge } \\
\text { Pariente }\end{array}$} & \multicolumn{2}{|c|}{$\begin{array}{l}\text { Cónyuge } \\
\text { Pariente } \\
\text { Cónyuge }\end{array}$} \\
\hline & & 0 & q & 0 & 우 & o & 9 & o. & \% & 0 & 우 & 0 & क & 0 & q & $\delta$ & 우 \\
\hline+4 & $\begin{array}{l}8 \\
q\end{array}$ & 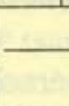 & -4 & $8 \mathrm{E}$ of. & $49 \mathrm{E}$ ? & & & & & & & & & & & & \\
\hline+3 & $\begin{array}{l}0 \\
+\end{array}$ & 10 & -3 & $8 \mathrm{E} \delta^{7}$. & 39E + & & & & & & & & & & & & \\
\hline+2 & $\begin{array}{l}0 \\
9\end{array}$ & 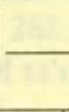 & -1 & 6E $\delta^{\circ}$. & $17 \mathrm{E}$ 우 & & & & & & & & & & & & \\
\hline+1 & $\begin{array}{l}0 \\
+\end{array}$ & $\begin{array}{l}1 E \\
2 E\end{array}$ & $\begin{array}{l}3 \mathrm{E} \\
4 \mathrm{E}\end{array}$ & 10 & $19 E$ & $18 \mathrm{E}$ & $28 \mathrm{E}$ & $23 \mathrm{E}$ & $24 \mathrm{E}$ & \begin{tabular}{|}
$33 \mathrm{E}$ \\
$34 \mathrm{E}$
\end{tabular} & $\begin{array}{l}35 \mathrm{E} \\
36 \mathrm{E}\end{array}$ & & & & & & \\
\hline . & $\begin{array}{l}0 \\
+9\end{array}$ & $\begin{array}{c}11 \mathrm{E} \\
14 \mathrm{E} \\
15 \mathrm{E}\end{array}$ & $x$ & $\begin{array}{l}11 \mathrm{E} \\
15 \mathrm{E}\end{array}$ & 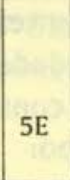 & $\begin{array}{c}11 \mathrm{E} \\
15 \mathrm{E}\end{array}$ & $5 \mathrm{E}$ & - & 29E & $32 \mathrm{E}$ ? & $q-$ & - & $\begin{array}{l}13 \mathrm{E} \\
0 \mathrm{E}-\mathrm{E} \\
12 \mathrm{E}\end{array}$ & $\begin{array}{l}44 \mathrm{E} \\
41 \mathrm{E} \\
47 \mathrm{E}\end{array}$ & $\begin{array}{l}37 \mathrm{E} \\
42 \mathrm{E}\end{array}$ & $\begin{array}{l}45 \mathrm{E} \\
46 \mathrm{E} \\
30 \mathrm{E}\end{array}$ & $\begin{array}{l}31 \mathrm{E} \\
43 \mathrm{E}\end{array}$ \\
\hline-1 & $\begin{array}{l}0 \\
+\end{array}$ & $\begin{array}{r}-8 \mathrm{E} \\
6 \mathrm{E}\end{array}$ & $\begin{array}{l}9 \mathrm{E}- \\
7 \mathrm{E}\end{array}$ & 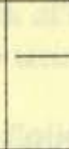 & -20 & $\mathrm{E}$ & & $33 \mathrm{E}$ & $22 \mathrm{E}$ & $25 \mathrm{E}$ & $26 \mathrm{E}$ & & & & waty & & \\
\hline-2 & $\begin{array}{l}0 \\
+\end{array}$ & & & $-21 \mathrm{E}$ & & & & & ef & & & & & & & & \\
\hline-3 & $\begin{array}{l}\sigma^{\circ} \\
q\end{array}$ & 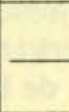 & (n) & $-40 \mathrm{E}$ & 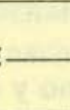 & & $\sqrt{2+3}$ & & III & & & & & & & & \\
\hline-4 & $\begin{array}{l}0 \\
q\end{array}$ & 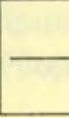 & & $-50 \mathrm{E}$ & - & & & & & & & & & & & & \\
\hline
\end{tabular}

Todo lo anterior se expresa en el diagrama 5 , donde se observa lo siguiente:

a. El sistema tiene nueve generaciones.

b. Tiene el mayor número de lexemas (50).

c. Hay un término recíproco para el suegro y el yerno (33E iyaam).

d. El término $14 \mathrm{E}$ at miim nombra al hermano materno de ego masculino. Solamente en este sistema se identificó esta designación, pues en 'Tapia Zenteno' el término 15B. ơ $\mathrm{Ho}>$, hoHo(a) $\mathrm{P}(\mathrm{M})$ at mim nombra al hermano mayor y al primo, de hombre.

e. A los consuegros se les nombra con préstamos de términos españoles correspondientes al parentesco ritual, antepuestos a un término huasteco (41E kumpaale atsii, 42E kumaale atsii).

De acuerdo con la tipología de Lowie es colateral bifurcado porque cada pariente recibe un término diferente:

P IE taata 2 2E pay'loom $\neq$

HoP 27E ts'uts' pay'loom $\neq$

HoM 18E itsaan

Con la tipología de Murdock, el sistema es hawaiano ya que con el mismo lexema se designa a las tres parientes:

Ha 5 E ebchal $=$

$\mathrm{Pr} / / 5 \mathrm{E}$ ebchal $=$

PrX 5E ebchal

Estudios de Cultura Maya. Vol. XXIII, 2003

Instituto de Investigaciones Filológicas/

Centro de Estudios Mayas, UNAM 
Designador sexual (7)

24F. $\delta^{*} \mathrm{HoEa}, \mathrm{EoHa}$ baay "cuñado de hombre", 25F. $+\mathrm{HaEo}$,EaHo jawan "cuñada de mujer", 26F. ơ HoP, ơ PEa pay'loom "tío paterno y suegro, de hombre", 27F. ㅇ HoP, $q$ PEo paap "tío paterno y suegro, de mujer"

Derivativo

28F. $\sigma^{\wedge} \mathrm{HaEa}, \delta^{\wedge} \mathrm{EaHo}$, $q \mathrm{HoE} o$, $q$ EoHa, $q$ EoHaEo at muul "cuñada de hombre, cuñado y concuño, de mujer"

Descriptivos

29F. ơ EoM pay'loom taata "padrastro de hombre", 30F. \& EoM paap taata "padrastro de mujer"

Términos terciarios (12)

Puros (6)

31F. PEo(a)ho(a) kumpaale "consuegro", 32F. MEo(a)ho(a) kumaale "consuegra"

Derivativos

33F. $\mathrm{PP}(\mathrm{M}) \mathrm{P}(\mathrm{M})$ chaabil pulek taata "bisabuelo", 34F. MP(M)P(M) chaabil pulek naana "bisabuela", 35F. ho(a)ho(a)ho(a) chaabil momob "bisnieto/a", 36F. haEo(a)P(M), 우 hoEo(a)P(M) chab kidhaab "hermanastra, hermanastro de mujer"

Designador sexual (6)

37F. + hoHo(a)P(M) ch'uuch' "primo de mujer", 38F. ơ haHo(a)P(M), ơ EaHoEa ixaam "prima y concuña, de hombre", 39F. $q$ haHo(a)P(M), $q$ EaHoEo bayil "prima y concuña, de mujer"

Derivativos

40F. $\delta^{*}$ EoHaEa at iya "concuño de hombre", 41F. \& EaHoEo at al'iib "concuña de mujer", 42F. ô hoEo(a)P(M) chab ataal "hermanastro de hombre"

Términos cuaternarios (2)

Puros (2)

Derivativos

43F. $\mathrm{P}(\mathrm{M}) \mathrm{P}(\mathrm{M}) \mathrm{P}(\mathrm{M}) \mathrm{P}(\mathrm{M})$ oxil pulek "tatarabuelo/a", 44F. ho(a)ho(a) ho(a)ho(a) oxil momob "tataranieto/a"

De la lista anterior se puede concluir que hay 23 términos elementales, 16 derivativos, tres descriptivos y dos préstamos del parentesco ritual español.

Aplicando el análisis componencial se tienen los siguientes rasgos: generación, línea directa, colateral paterno, colateral materno, afinidad (por los cónyuges, por los parientes del cónyuge, por los cónyuges de los parientes, por los parientes de los cónyuges de 
los parientes y por los cónyuges de los parientes de los cónyuges), el sexo del hablante y el sexo del pariente. El diagrama 6 permite obtener una amplia visión del sistema.

Lo que ahora procede es clasificar el sistema que, según Lowie, es del tipo colateral bifurcado, igual que los otros sistemas exceptuando 'Siglo xvi' y 'Chicomucelteco':

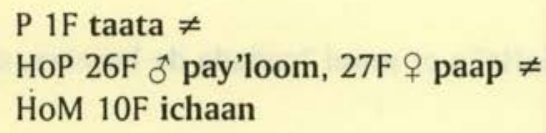

De acuerdo con la clasificación de Murdock, este sistema es diferente de 'Tamaletón' y 'Siloxúchil' ya que pertenece al tipo esquimal, coincidiendo en esto con el 'Chicomucelteco':

$$
\begin{aligned}
& \text { Ego } \delta: \text { Ha } 8 \mathrm{~F} \text { kidhaab } \neq \\
& \operatorname{Pr} / / 38 \mathrm{~F} \text { ixaam }= \\
& \operatorname{PrX} 38 \mathrm{~F} \text { ixaam } \\
& \text { Ego } 9: \text { Ha } 8 \mathrm{~F} \text { kidhaab } \neq \\
& \operatorname{Pr} / / 39 \mathrm{~F} \text { bayil }= \\
& \operatorname{PrX} 39 \mathrm{~F} \text { bayil }
\end{aligned}
$$

VII. Sistema de parentesco huasteco de San Francisco, Veracruz

La población hablante de huasteco en el municipio de Chontla es de 2,867 y San Francisco es una de las comunidades de ese municipio. Se describen los 37 términos de parentesco que se obtuvieron en el trabajo de campo:

Términos primarios $(10)$

Puros (8)

1G. P paap "padre", 2G. P paapa "padre", 3G. M maama "madre", 4G.

$\mathrm{Ho}(\mathrm{a}), \mathrm{ho}(\mathrm{a}) \mathrm{Ho}(\mathrm{a}) \mathrm{P}(\mathrm{M})$ ebal "hermano/a,primo/a", 5G. ho kwitoolil, "hijo", 6G. ho(a) chikaamil "hijo/a", 7G. ha chidhaanil "hija", 8G. Eo(a) tomkiil "esposo/a"

Designador sexual (2)

9G. + Eo yelchelel "esposo", 10G. ơ Ea ixal "esposa"

\section{Términos secundarios (20)}

Puros (13)

11G. PP(M) maam "abuelo", 12G. MP(M) aach "abuela", 13G. HoP(M) ichaan "tío", 14G. HaP(M) chanuub "tía", 15G. ho(a)Ho(a) ichak' "sobrino/a", 16G. ho(a)ho(a) momob "nieto/a", 17G. Eaho al'iib "nuera", 18G. EoHa,HoEo(a), ๆ EoHaEo baay "cuñado, concuño de mujer"

Derivativos

19G.PP(M) pulik paap "abuelo", 20G.MP(M) pulik ma "abuela”, 21G. EoM paple' "padrastro", 22G.EaP mimle' "madrastra", 23G.ho(a)Eo(a) t'amle' "hijastro/a" 
Diagrama 6. Análisis componencial de la terminología de parentesco del huasteco de Loma Larga, Veracruz

\begin{tabular}{|c|c|c|c|c|c|c|c|c|c|c|c|c|c|c|c|c|c|}
\hline \multirow[t]{2}{*}{ G } & \multirow[t]{2}{*}{$\begin{array}{l}E \\
G \\
O\end{array}$} & \multicolumn{2}{|c|}{$\begin{array}{c}\text { Línea } \\
\text { Directa }\end{array}$} & \multicolumn{2}{|c|}{$\begin{array}{l}\text { Colateral } \\
\text { Paterno }\end{array}$} & \multicolumn{2}{|c|}{$\begin{array}{l}\text { Colateral } \\
\text { Materno }\end{array}$} & \multicolumn{2}{|c|}{$\begin{array}{c}\text { Cónyuge } \\
\text { del } \\
\text { Pariente }\end{array}$} & \multicolumn{2}{|c|}{$\begin{array}{c}\text { Pariente } \\
\text { del } \\
\text { Cónyuge }\end{array}$} & \multicolumn{2}{|c|}{ Cónyuge } & \multicolumn{2}{|c|}{$\begin{array}{l}\text { Pariente } \\
\text { Cónyuge } \\
\text { Pariente }\end{array}$} & \multicolumn{2}{|c|}{$\begin{array}{l}\text { Cónyuge } \\
\text { Pariente } \\
\text { Cónyuge }\end{array}$} \\
\hline & & 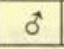 & q & 0 & ㅇ & $\sigma$ & q & 0 & q & 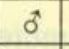 & ㅇ & 0 & 우 & 0 & q & 0 & $q$ \\
\hline+4 & $\begin{array}{l}\text { के } \\
+\end{array}$ & 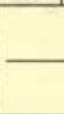 & 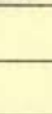 & $-43 F$ & & & & & & & & & & & & & \\
\hline+3 & oै & 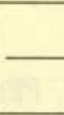 & -3 & F 0.3 & $4 \mathrm{~F}$ ㅇ- & 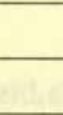 & & & & & & & & & & & \\
\hline+2 & $\begin{array}{l}0 \\
+ \\
\end{array}$ & 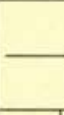 & -1 & $F 0^{*}, 1$ & $3 \mathrm{~F}$ ㅇ․ & & & & & & & & & & & & \\
\hline+1 & 우 & $1 \mathrm{~F}$ & $2 \mathrm{~F}$ & $\begin{array}{l}26 \mathrm{~F} \\
27 \mathrm{~F} \\
\end{array}$ & $11 \mathrm{~F}$ & $10 \mathrm{~F}$ & $12 \mathrm{~F}$ & $\begin{array}{l}29 \mathrm{~F} \\
19 \mathrm{~F} \\
30 \mathrm{~F}\end{array}$ & $\begin{array}{l}20 \mathrm{~F} \\
23 \mathrm{~F} \\
27 \mathrm{~F} \\
\end{array}$ & $26 \mathrm{~F}$ & $12 \mathrm{~F}$ & & & & & & \\
\hline 0 & 8 & $\begin{array}{l}9 \mathrm{~F} \\
8 \mathrm{~F}\end{array}$ & $8 \mathrm{~F}$ & 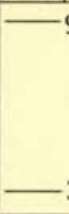 & F 0. & $38 \mathrm{~F}$ \% & 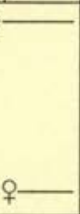 & $\overline{-}$ & $24 \mathrm{~F}$ o & $28 \mathrm{~F}$ & क- & & $7 \mathrm{~F}-$ & $\begin{array}{l}42 \mathrm{~F} \\
31 \mathrm{~F} \\
36 \mathrm{~F}\end{array}$ & $\begin{array}{l}32 \mathrm{~F} \\
36 \mathrm{~F}\end{array}$ & $\begin{array}{c}9 \mathrm{~F} \\
40 \mathrm{~F} \\
28 \mathrm{~F}\end{array}$ & $\begin{array}{l}38 \mathrm{~F} \\
41 \mathrm{~F} \\
39 \mathrm{~F}\end{array}$ \\
\hline-1 & $\sigma^{5}$ & $\begin{array}{r}3 \mathrm{~F} \\
-5\end{array}$ & $=-\frac{}{4 \mathrm{~F}}$ & 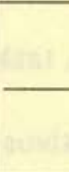 & -13 & & & $15 \mathrm{~F}$ & $16 \mathrm{~F}$ & $21 \mathrm{~F}$ & $22 \mathrm{~F}$ & & & & & & $\sqrt{4} 3$ \\
\hline-2 & के & & - & -14 & & & & & & & & & & & & & 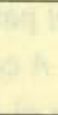 \\
\hline-3 & $\begin{array}{l}6 \\
+\end{array}$ & & - & -35 & 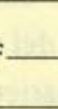 & & & & & & & & & & & & \\
\hline-4 & $\begin{array}{l}0 \\
\text { 우 }\end{array}$ & & - & & & & & & & & & & & & & & \\
\hline
\end{tabular}

El diagrama permite observar los siguientes aspectos relevantes:

a. Consta de nueve generaciones.

b. Designa con términos especiales al hermano del padre pero en este caso, a diferencia del sistema potosino y del de 'Siloxúchil', nombra además al suegro, pariente afín, y expresa la categoria sexo del hablante (26F \& pay'loom, 27F $q$ paap). Misma situación se presenta con el término que nombra a la hermana de la madre (12F miim), porque se refiere también a la suegra, sin considerar la categoría sexo del hablante.

c. Los tatarabuelos se denominan con un lexema (43F oxil pulek) como lo hace 'San Francisco', cosa que no sucede en el sistema potosino que tiene un vocablo para cada pariente.

d. Este sistema tiene términos consanguíneo-afines, como ya se ha visto en el inciso b. Pero a ésos hay que agregar los siguientes: $9 \mathrm{~F}$ ơ ataal 'hermano, primo, concuño', 38F đ̊ ixaam 'prima, concuña' y 39F $q$ bayil 'prima, concuña'.

e. Como se comentó en 'Siloxúchil', en este sistema también los cunados se designan con tres términos, $24 \mathrm{~F} \delta$ baay 'cuñado' y $25 \mathrm{~F}$ q jawan 'cuñada' se refieren al pariente de su mismo sexo y con otro, $28 \mathrm{~F}$ at mul, ambos egos nombran al pariente de sexo opuesto.

f. En lo que se refiere a las primas, se tienen términos especificos que, además, expresan la categoría sexo del hablante (38F $\delta$ ixaam, $39 \mathrm{~F} \&$ bayil).

g. Tres vocablos nombran al padrastro, uno derivativo (19F chab taata) y dos descriptivos de acuerdo con el sexo de ego (26F $\delta$ pay'loom taata, $27 \mathrm{~F} \&$ paap taata).

h. No hay términos recíprocos.

i. Ego femenino designa al hermano y a la hermana con la forma $8 \mathrm{~F}$ kidhaab.

j. Su terminología es la más diversificada de los cuatro sistemas analizados en esta ocasión.

Estudios de Cultura Maya. Vol. XXIII, 2003

Instituto de Investigaciones Filológicas/

Centro de Estudios Mayas, UNAM

ISSN 0185-2574 
Designador sexual (7)

24G. $\delta^{\star} \mathrm{PEa}$,Eoha iyaam "suegro de hombre, yerno", 25G. $\delta^{\wedge} \mathrm{EoHa}, \delta^{\wedge} \mathrm{HoEa}, \delta^{\star} \mathrm{EoHaEa}$ ch'uuch' "cuñado y concuño, de hombre", 26G. $\delta^{\top}$ EaHo, $\delta^{\wedge} \mathrm{HaEa}, \delta^{\top}$ EaHoEa ixaam "cuñada y concuña, de hombre", 27G. ㅇ EaHo, $\uparrow$ HaEo, + EaHoEo jawan "cuñada y concuña, de mujer"

\section{Derivativos}

28G. ㅇ PEo inik al'iib "suegro de mujer", 29G. $\sigma^{\wedge}$ MEa uxum iyaam "suegra de hombre", 30G. + MEo uxum al'iib "suegra de mujer"

\section{Términos terciarios (6)}

Puros (5)

31G.P(M)P(M)P(M),ho(a)ho(a)ho(a) chaabil "bisabuelo/a,bisnieto/a”, 32G. PEo(a)ho(a) kumpaale "consuegro", 33G. MEo(a)ho(a) kumaale "consuegra"

\section{Derivativos}

34G. ho(a)Eo(a)P(M) ch'ejel ebal "hermanastro/a", 35G. ho(a)Eo(a)P (M) pulik ebal "hermanastro/a"

\section{Designador sexual (1)}

Derivativo

36G. đิ EoHaEa at iya "concuño de hombre"

\section{Términos cuaternarios (1)}

Puros (1)

37G. $P(M) P(M) P(M) P(M)$, ho(a)ho(a)ho(a)ho(a) oxil "tatarabuelo/a, tataranieto/a"

Esta relación comprende 24 términos elementales, 11 derivativos y dos préstamos del parentesco ritual español.

A continuación se verá qué rasgos pertinentes están presentes en el material, para aplicar el análisis componencial de Goodenough: generación, línea directa, colateral paterno, colateral materno, afinidad (por los cónyuges, por los parientes del cónyuge, por los cónyuges de los parientes, por los parientes de los cónyuges de los parientes y por los cónyuges de los parientes de los cónyuges), el sexo del hablante y el sexo del pariente. En el diagrama 7 se muestra lo anterior.

Algunos comentarios finales sobre las terminologías de parentesco, relacionados con los términos derivativos (Murdock, 1965: 98), permiten observar en algunos sistemas los siguientes elementos: tzutzu tzutz ts'uts' "pequeño", uxum "mujer", tzabil tsabil "numeral dos tsab, más el sufijo de relación genitiva -il" , pulék pulek pulik "grande", chab "numeral dos", inik "hombre", chaabil "numeral dos chaab, más el sufijo de relación genitiva -il”, oxil "numeral tres ox, más el sufijo de relación genitiva -il”, ch'ejel "viejo".

Los términos, préstamos del español, paapa, maama, kumpaale y kumaale que designan al padre, la madre, el consuegro y la consuegra respectivamente, se obtuvieron tal como se transcribieron. Con respecto a los dos últimos parientes, Tapia Zenteno 
Diagrama 7. Análisis componencial de la terminología de parentesco del huasteco de San Francisco, Veracruz

\begin{tabular}{|c|c|c|c|c|c|c|c|c|c|c|c|c|c|c|c|c|c|}
\hline \multirow[t]{2}{*}{ G } & \multirow[t]{2}{*}{$\begin{array}{l}E \\
G \\
O\end{array}$} & \multicolumn{2}{|c|}{$\begin{array}{c}\text { Línea } \\
\text { Directa }\end{array}$} & \multicolumn{2}{|c|}{$\begin{array}{l}\text { Colateral } \\
\text { Paterno }\end{array}$} & \multicolumn{2}{|c|}{$\begin{array}{l}\text { Colateral } \\
\text { Materno }\end{array}$} & \multicolumn{2}{|c|}{$\begin{array}{c}\text { Cónyuge } \\
\text { del } \\
\text { Pariente }\end{array}$} & \multicolumn{2}{|c|}{$\begin{array}{c}\text { Pariente } \\
\text { del } \\
\text { Cónyuge }\end{array}$} & \multicolumn{2}{|c|}{ Cónyuge } & \multicolumn{2}{|c|}{$\begin{array}{l}\text { Pariente } \\
\text { Cónyuge } \\
\text { Pariente }\end{array}$} & \multicolumn{2}{|c|}{$\begin{array}{l}\text { Cónyuge } \\
\text { Pariente } \\
\text { Cónyuge }\end{array}$} \\
\hline & & 0 & q & 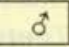 & $q$ & 0 & $q$ & 0 & q & 0 & 웅 & $\sigma^{\circ}$ & 웅 & 8 & 우 & $\hat{\sigma}$ & q \\
\hline+4 & o & 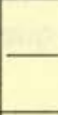 & - & -37 & & & & & & & & & & & & & \\
\hline+3 & $\begin{array}{l}\text { क } \\
\text { क }\end{array}$ & 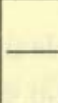 & - & -31 & & & & & & & & & & & & & \\
\hline+2 & $\begin{array}{l}0 \\
+ \\
+\end{array}$ & 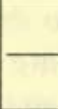 & $11 \mathrm{G}$, & 19G ठ & $12 \mathrm{G}, 2$ & $20 \mathrm{G}$ & & & & & & & & & & & \\
\hline+1 & $\begin{array}{l}0 \\
+\end{array}$ & $\begin{array}{l}1 G \\
2 G\end{array}$ & $3 G$ & -1 & G o $\sigma^{*}$. & $14 \mathrm{G}$ & - & $21 \mathrm{G}$ & $22 \mathrm{G}$ & $\begin{array}{l}24 G \\
28 G\end{array}$ & $\begin{array}{l}29 G \\
30 G\end{array}$ & & & & & & \\
\hline 0 & 우 & 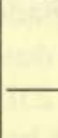 & 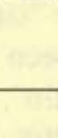 & -4 & & & & $\begin{array}{l}25 G \\
18 G\end{array}$ & $26 \mathrm{G}$ & $\begin{array}{l}25 G \\
18 G\end{array}$ & $\begin{array}{l}26 \mathrm{G} \\
27 \mathrm{G}\end{array}$ & $\overline{9 G}$ & $8 \mathrm{G}-$ & $\begin{array}{l}32 \mathrm{G} \\
-34 \mathrm{G}\end{array}$ & $\begin{array}{l}33 G \\
35 G\end{array}$ & $\begin{array}{r}25 \mathrm{G} \\
36 \mathrm{G} \\
18 \mathrm{G}\end{array}$ & $\begin{array}{l}26 G \\
27 G\end{array}$ \\
\hline-1 & 우 & $\begin{array}{l}5 G \\
-6\end{array}$ & $\mathrm{G}$ & 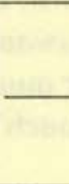 & -15 & & & $24 \mathrm{G}$ & $17 \mathrm{G}$ & -23 & b- & & & & & & \\
\hline-2 & $\begin{array}{l}\text { t } \\
+\end{array}$ & & - & & & & & & W & & & & & & & & \\
\hline-3 & $\begin{array}{l}\sigma^{\circ} \\
\text { क }\end{array}$ & & - & -3 & & & & & & & & & & & & & \\
\hline-4 & $\begin{array}{l}\sigma^{\circ} \\
+\end{array}$ & & 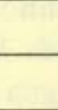 & -3 & & & & & & & & & & & & & \\
\hline
\end{tabular}

Aspectos relevantes que conviene señalar:

a. Aunque tiene nueve generaciones como en los otros sistemas, los términos de las G3 (31G chaabil) y G4 (37G oxil) son recíprocos de las G-3 y G-4 respectivamente. En este caso solamente quedan los numerales dos (chaabil) y tres (oxil), porque los términos de parentesco han desaparecido.

b. El término $18 \mathrm{G}$ baay designa al cuñado de ambos egos. Este término en los otros sistemas es usado solamente por el hombre.

c. Los hermanastros se nombran con $34 \mathrm{C}$ ch'ejel ebal $\sim 35 \mathrm{C}$ pulik ebal (en el sistema potosino $34 \mathrm{G}$ designa al medio hermano) y los hijastros con $23 \mathrm{G}$ t'amle', siendo ambos términos genéricos, mientras que en los otros sistemas se tienen lexemas específicos.

d. A diferencia de los otros dialectos, no hay vocablo especial para el hermano del padre ni para la hermana de la madre.

e. No tiene términos descriptivos y cuenta con dos préstamos del parentesco ritual español para nombrar a los consuegros.

Según Lowie, el sistema es tipo lineal ya que designa con un término al padre y con otro término a los dos tíos:

$P$

HoP $13 \mathrm{G}$ ichaan=

HoM $13 \mathrm{G}$ ichaan

y considerando la propuesta de Murdock, es tipo hawaiano porque a las tres parientes las nombra con el mismo término:

$\mathrm{Ha} 4 \mathrm{G}$ ebal $=$

$\mathrm{Pr} / / 4 \mathrm{G}$ ebal $=$

$\operatorname{PrX} 4 \mathrm{G}$ ebal

Estudios de Cultura Maya. Vol. XXIII, 2003

Instituto de Investigaciones Filológicas/

Centro de Estudios Mayas, UNAM

ISSN 0185-2574 
proporciona otros términos, 41B.PEo(a)ho(a) yetzel atzii y 46B.MEo(a)ho(a) uxum atzii, pero para el compadre y la comadre registra compalle y comalle. Si bien este autor no reporta vocales largas en el resto del material de parentesco en estudio, excepto el término atzii, resulta interesante considerar lo que señala Ochoa (1995: 125 ) en su estudio de un documento huasteco del siglo xvi. Explica que, en algunos casos, parece que la longitud vocálica se indica duplicando la consonante vecina a la vocal. Si se aplicara este criterio al material lingüístico de Tapia Zenteno, entonces los términos serían compaale y comaale, lexemas cognados de forma con los que se obtuvieron para los consuegros en 'Tamaletón', 'Loma Larga' y ‘San Francisco'.

VIII. Aquí corresponde hacer una reflexión sobre la terminología que designa a los cuñados y para ello conviene comentar la postura de Lévi-Strauss (1969: 45) quien afirma que: “[...] la relación de los 'cuñados' forma el eje inevitable en torno del cual se construye la estructura de parentesco". De esta manera modifica radicalmente la estructura de la familia con su propuesta del 'átomo del parentesco', donde ya aparece la figura del cuñado, puesto que un hombre solamente puede obtener una mujer de manos de otro hombre, el cual la cede bajo la forma de hija o de hermana.

Se observa en los sistemas de este estudio (cuadro 4) exceptuando 'Siglo xv', que 'Tapia Zenteno' dispone de tres términos para designarlos, 30B ô bay "cuñado", 31B + xahuan "cuñada", 40B at mul + "cuñado" y $\widehat{o}$ "cuñada"; 'Chicomucelteco' dos, $14 \mathrm{C}$ úai "cuñado" y $16 \mathrm{C}$ atmún "cuñada"; 'Siloxúchil' tres, 22D ơ bayj "cuñado", 23D $q$ jauán

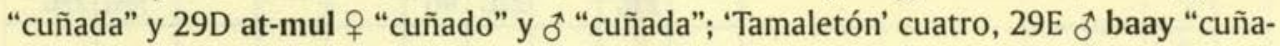
do", $30 \mathrm{E}+$ xibaam "cuñado", $31 \mathrm{E} \delta$ ixaam "cuñada" y $32 \mathrm{E}+$ jawan "cuñada"; "Loma Larga' tres, $24 \mathrm{~F} \delta^{\top}$ baay "cuñado", $25 \mathrm{~F} q$ jawan "cuñada" y $28 \mathrm{~F}$ at muul $q$ "cuñado" y $\sigma^{\star}$ “cuñada” y 'San Francisco' cuatro, 18G baay “cuñado”, 25G ôt ch'uuch' “cuñado”, 26G ô ixaam "cuñada", $27 \mathrm{G}$ + jawan "cuñada".

Con objeto de conocer qué lexemas y cuántos nombran a los cuñados en algunas lenguas de la familia maya, se presentan en el cuadro 2 datos del siglo xvi y datos más recientes, donde se tiene, en el tojolabal (Ruz, 1982: 159-161), un término que emplea ego masculino para designar al cuñado y a la cuñada (baluch) posponiéndole los lexemas winik "hombre" e ixuk "mujer", ya que el resto de los términos pertenece a parientes consanguíneos; el chortí (Wisdom, 1961: 300) emplea el término mu' para todos los cuñados; el maya-yucateco de Hubilá (Anzaldo y Briceño, 1998: 205) mantiene el término para el cuñado, baal, y para la cuñada emplea el que designa a la hermana mayor kiik prefijándole el numeral dos ka'; los sistemas del maya-yucateco del siglo xvı (Borodatova y Kozhanovskaya, 1999: 335), tzeltal de Tenejapa (Medina, 1992: 119), tzotzil de San Pablo Chalchihuitán (Guiteras, 1966: 166) y maya-mopán (Schumann, 1997: 36), cuentan con los mismos tres términos, bal jbol, mu' jmu' y jawan jabän. El tzotzil de San Andrés (Hurley y Ruiz, 1978: 280), además de los tres términos de los sistemas anteriores, presenta un elemento diferente, yit'ix, para el cuñado de ego masculino, aumentando a cuatro el número de lexemas; en cuanto al ch'ol (Esponda, 1996: 109), se observa que para designar al cuñado tiene cinco términos, uno de los cuales, mu', es común en varios sistemas. La cuñada de ego femenino se designa con el lexema jawan, ascendiendo de esta manera el número de términos a seis. En el caso del cakchiquel (Blair, 1981: 480-481) y el pokomam del siglo xvi (Miles, 1983: 81-82), designan a 


\section{Cuadro 2. Términos que designan a los cuñados en algunas lenguas mayances}

\begin{tabular}{|c|c|c|c|c|}
\hline \multirow[t]{2}{*}{ Lengua } & \multicolumn{2}{|c|}{ Cuñado } & \multicolumn{2}{|c|}{ Cuñada } \\
\hline & 8 & 웅 & 8 & 운 \\
\hline \multirow{4}{*}{$\begin{array}{l}\text { tojolabal } \\
\text { (Ruz, 1982: } \\
\text { 159-161) }\end{array}$} & \multicolumn{2}{|c|}{ mayores que ego } & \multicolumn{2}{|c|}{ mayores que ego } \\
\hline & $\begin{array}{l}\text { baluch winik } \\
\text { bank'il* }\end{array}$ & nu" & $\begin{array}{l}\text { baluch ixuk } \\
\text { watz }\end{array}$ & watz ${ }^{*}$ \\
\hline & \multirow{2}{*}{\multicolumn{2}{|c|}{$\begin{array}{l}\text { menores que ego } \\
\text { ijtz'in** }\end{array}$}} & \multirow{2}{*}{\multicolumn{2}{|c|}{$\begin{array}{l}\text { menores que ego } \\
\text { ijtz'in** }\end{array}$}} \\
\hline & & & & \\
\hline $\begin{array}{l}\text { chortí } \\
\text { (Wisdom, 1961: } \\
\text { 300) }\end{array}$ & mu' & $\mathrm{mu}$ & $\mathrm{mu} \mathbf{u}^{\prime}$ & $\mathrm{mu}^{\prime}$ \\
\hline $\begin{array}{l}\text { maya yucateco } \\
\text { de Hubilá } \\
\text { (Anzaldo y } \\
\text { Briceño, 1998: } \\
\text { 205) }\end{array}$ & baal & baal & ka'kiik & ka'kiik \\
\hline $\begin{array}{l}\text { maya-yucateco } \\
\text { del siglo xvı } \\
\text { (Borodatova y } \\
\text { Kozhanovskaya } \\
\text { 1999: } 335 \text { ) }\end{array}$ & baal & muu & muu & hawan \\
\hline $\begin{array}{l}\text { tzeltal de Tenejapa } \\
\text { (Medina, 1992: } \\
\text { 119) }\end{array}$ & bal & mu' & $\mathrm{mu}^{\prime}$ & jawan \\
\hline $\begin{array}{l}\text { tzotzil de San Pablo } \\
\text { Chalchihuitán } \\
\text { (Guiteras, 1966: } \\
\text { 166) }\end{array}$ & jbol & jmu' & jmu’ & jawan \\
\hline $\begin{array}{l}\text { maya mopán } \\
\text { (Schumann, } \\
\text { 1997: 36) }\end{array}$ & bal & mu' & $\sqrt{1}+2$ & jabān \\
\hline $\begin{array}{l}\text { tzotzil de San } \\
\text { Andrés } \\
\text { (Hurley y Ruíz, } \\
\text { 1978: 280) }\end{array}$ & sbol & smu' & yit'ix & sjavan \\
\hline $\begin{array}{l}\text { ch'ol de Tila y } \\
\text { Salto de Agua } \\
\text { (Esponda, 1996: } \\
\text { 109) }\end{array}$ & $\begin{array}{l}\text { ja'an } \\
\text { askun } \\
\text { ku } \\
\text { ku'un }\end{array}$ & $\begin{array}{l}\text { mu' } \\
\text { askun } \\
\text { ku } \\
\text { ku'un }\end{array}$ & $\mathrm{mu}^{\prime}$ & jawan \\
\hline $\begin{array}{l}\text { cakchiquel } \\
\text { (Blair, 1981: } \\
\text { 480-481) }\end{array}$ & baluc & echa'n & ixnan & ali' \\
\hline $\begin{array}{l}\text { pokomam del } \\
\text { siglo xvı } \\
\text { (Miles, 1983: } \\
81-82 \text { ) }\end{array}$ & $\begin{array}{l}\text { baluk } \\
\text { HoEa }\end{array}$ & $\begin{array}{l}\text { echam } \\
\text { icham } \\
\text { HoEo }\end{array}$ & $\begin{array}{l}\text { ixnam } \\
\text { HaEa }\end{array}$ & $\begin{array}{l}\text { ilim } \\
\text { HaEo }\end{array}$ \\
\hline
\end{tabular}

- Términos consanguíneos que designan a los hermanos mayores

* Término consanguíneo que designa a los hermanos menores

Estudios de Cultura Maya. Vol. XXIII, 2003

Instituto de Investigaciones Filológicas/

Centro de Estudios Mayas, UNAM

ISSN 0185-2574 
los cuñados con cuatro términos diferentes de los anteriores. Sobre el lexema del cuñado de ego masculino conviene expresar que es el mismo que emplea ego masculino en la terminología de parentesco del tojolabal.

Resulta evidente la estabilidad genética del término que designa a la cuñada de ego femenino, tanto en los sistemas de la rama huastecana como en varias otras lenguas de la familia maya. Con respecto a la terminología que corresponde al cuñado de ego masculino, así como al que designa a los parientes del sexo opuesto en la rama huastecana, baay y at muul, un estudio posterior sobre procesos morfológicos o de otra índole permitirá establecer si tienen relación genética con los lexemas baal, bol, baluc baluch el primero y con me' el segundo.

IX. Una vez realizadas la descripción y clasificación de los siete sistemas de parentesco de la rama huastecana, el paso siguiente es comparar primero sus estructuras representadas en los diagramas $1,2,3,4,5,6$ y 7 , con objeto de observar qué rasgos comparten, en cuáles difieren, cómo se manifiestan las categorías de Kroeber. En fin, qué aspectos importantes se pueden determinar, y posteriormente comparar las terminologías para establecer el número de cognadas de forma y significado, los desplazamientos semánticos y los lexemas sin cognación. A continuación se enlistan los aspectos que son relevantes:

\section{Las estructuras}

1. Tendencia de las descripciones modernas a proporcionar términos para más generaciones, llegando a cubrirse hasta nueve. En este caso no se trata de una innovación de la lengua, sino que se debe a la descripción hecha por los correspondientes investigadores.

2. La categoría marcada sexo del hablante, está presente en todos los sistemas del huasteco, lo que constituye un rasgo particular de esa lengua y que se observa principalmente en las generaciones centrales, $1,0 \mathrm{y}-1$, aunque en el caso de 'Tapia Zenteno' se extiende hasta la $\mathrm{G}^{-2}$. Con respecto al 'chicomucelteco', esa categoría solamente se expresa en los términos de los hijos y de los esposos.

3. En todos los sistemas de parentesco se expresan cinco categorías de Kroeber (generación, consanguíneo/afín, lineal/colateral, sexo del hablante y sexo del pariente). 'Tapia Zenteno' y 'Chicomucelteco' son los únicos sistemas que incluyen la categoría edad relativa dentro de una misma generación. 'Tamaletón' y 'Loma Larga' registran la categoría sexo del pariente que da la relación de parentesco y 'Tapia Zenteno' sólo lo hace por la línea paterna. En cambio en 'Chicomucelteco' y 'San Francisco' esa categoría está ausente. Ningún sistema documentó la categoría "condición de vida de la persona que relaciona".

4. 'Tapia Zenteno' consigna los términos de los concuños y de los consuegros, datos que no siempre se proporcionan. Esto hizo necesario añadir dos columnas a los diagramas usuales para insertar los lexemas correspondientes a esos parientes.

5. Es notable que, en los sistemas contemporáneos, los términos lineales de las $\mathrm{G}^{4}$, $\mathrm{G}^{3}, \mathrm{G}^{2}$ y sus correspondientes descendentes, se extienden a los colaterales, situación que no consideran los sistemas de 'Tapia Zenteno' y del 'Chicomucelteco' en las generaciones superiores a la $\mathrm{G}^{1} \mathrm{e}$ inferiores a la $\mathrm{G}^{-1}$. 
6. Se observa en la $\mathrm{G}^{\circ}$ que ego masculino se refiere a los primos varones con el mismo término con que nombra al hermano, mostrando una tendencia hawaianizante (Murdock establece el tipo hawaiano para la hermana y las primas). Dicha tendencia es extrema en 'San Francisco', donde tanto ego masculino como ego femenino designan al primo y a la prima (sean paralelos o cruzados) con el mismo término del hermano y de la hermana. Si bien 'Loma Larga' introduce cambios, esta característica es estable en el huasteco, pero no en el 'chicomucelteco', puesto que tiene vocablos diferentes para esos parientes.

7. Las descripciones modernas, a pesar de que son acuciosas, muestran reducción de distinciones en el tío paterno. 'Tapia Zenteno' proporciona una amplia terminología para ese pariente, probablemente porque se le concedía mayor importancia a la línea paterna.

8. Las estructuras de los cuñados son idénticas en 'Tapia Zenteno', 'Siloxúchil' y 'Loma Larga'; 'Tamaletón' muestra diferencias y 'San Francisco' introduce innovaciones.

9. En la estructura del sistema de 'Tapia Zenteno' está vacío el espacio correspondiente a la hija. Se desconoce por qué no están representadas las mujeres de la $\mathrm{G}^{-1}$ y también llama la atención el habla por sexos en la $\mathrm{G}^{-2}$.

10. 'Siloxúchil' es el único de los sistemas modernos que diferencia los sobrinos paralelos de los cruzados. Aunque 'Tapia Zenteno' solamente proporciona los sobrinos cruzados masculinos de ambos egos y no da información de los sobrinos paralelos, se observa esta misma distinción.

\section{Los sistemas terminológicos}

11. Las terminologías de los siete sistemas sumaron 241 lexemas. Se obtuvieron las siguientes cognadas: cuatro en cuatro conjuntos de seis sistemas; nueve en nueve conjuntos de cinco sistemas; seis en seis conjuntos de cuatro sistemas; 15 en 15 conjuntos de tres sistemas y 23 en 23 conjuntos de dos sistemas. Algunas cognadas presentaron desplazamientos. En total se tienen 57 cognadas. El cambio semántico se presentó en 55 términos que son cognados de forma y parcialmente de significado. 56 lexemas no tuvieron relación genética aparente. El número de cognadas de forma y significado correspondientes a términos consanguíneos supera al de cognadas de términos afinẹs y se localizan todas ellas, principalmente, en las generaciones 0 y descendentes, aunque también aparecen en las ascendentes, pero en número menor. Los préstamos del parentesco ritual español corresponden a los dialectos actuales y designan a los consuegros.

12. 'Siglo xv' es el único sistema que tiene el $100 \%$ de cognadas de forma y significado. El resto de los sistemas presentaron los siguientes porcentajes: 'Siloxúchil' $85 \%$, 'Loma Larga' 80\%, 'Tapia Zenteno' 78\%, 'San Francisco' 73\%, 'Tamaletón' 72\% y ‘Chicomucelteco' $59 \%$. No fue posible encontrar cognadas absolutas, debido principalmente a que la terminología del 'Siglo xvi' es limitada. Sin embargo, en los seis sistemas restantes se obtuvieron dos términos cognados de forma y significado: el que nombra al tío materno (itzan, ichán, itsaan, ichaan) término que en algunas lenguas de la familia maya tiene distintos referentes, ${ }^{25}$ y el que designa al cuñado de ego masculino (bay, úai,

\footnotetext{
${ }^{25}$ Véase la nota 18.
} 
bayj, baay). Sobre este particular, atendiendo a la propuesta de Lévi-Strauss, se realizó un estudio de los lexemas que nombran a los cuñados en algunas lenguas de la familia maya, observándose en este grupo que, con algunas excepciones, mantienen una terminología amplia.

13. En cuanto al resto de las cognadas que se presentaron en parte de los sistemas, en general se observa que 'Siglo xv', 'Tapia Zenteno' y 'Tamaletón' mantienen lazos más estrechos y 'Chicomucelteco' guarda escasa relación con todos los sistemas. Entre 'Siglo xv' por una parte y 'Siloxúchil' y 'Loma Larga' por otra, no se obtuvieron cognadas de forma y significado, sí las hubo de significado solamente. Hay un término kidhaab, 'hermano' en 'Siglo xv', que se desplaza en 'Siloxúchil' y 'Loma Larga', extendiéndose a la hermana.

Cabe también apuntar que este lexema solamente se presentó en estos tres sistemas y que en San Luis Potosí kidhaab es un elemento de la palabra "maldad", quith_btal_b (Larsen, 1955: 163).

14. Los desplazamientos quedaron distribuidos como se indica: 23 por extensión, 13 por sustitución, 15 por reducción, cuatro por reducción-extensión y uno por reducción-sustitución. El mayor número de cambios se presentó en los sistemas contemporáneos, especialmente en 'Tamaletón' y 'Loma Larga'. Hay dos términos cognados de forma, ch'uuch' y chakaamil tsakaamil cuyo desplazamiento no se puede determinar por pertenecer a sistemas actuales. El primero denomina en 'Siloxúchil' a 'hermano y primo' de ego femenino, a 'primo' también de ego femenino en 'Loma Larga' y a 'cuñado, concuño' de ego masculino en 'San Francisco'. El segundo término designa en 'Tamaletón', 'Loma Larga' y 'San Francisco' a ambos hijos, pero en 'Siloxúchil' se refiere además a los sobrinos paralelos de ambos egos. En 'Loma Larga' los cambios semánticos por sustitución dan lugar a que los términos consanguíneos designen no sólo a parientes de esa categoría, sino también a los de la afín. 'San Francisco' realiza más desplazamientos extensionistas que, como se señaló anteriormente, producen características hawaianizantes.

15. 'Tamaletón' es el más conservador, le sigue 'Siloxúchil', 'San Francisco' se encuentra en estado de transición y 'Loma Larga' resulta ser, indudablemente, el más dinámico. De los tres sistemas de Veracruz, 'Siloxúchil y 'Loma Larga' guardan más relación. En cambio 'San Francisco' en el aspecto terminológico muestra semejanzas con 'Tamaletón'.

16. La estabilidad genética de los términos da los siguientes porcentajes:

Porcentaje de cognadas absolutas: 0

Porcentaje de cognadas parciales: $129 / 241=0.54$

Porcentaje de desplazamientos: $55 / 241=0.23$

Porcentaje de lexemas sin cognación: $57 / 241=0.23$

\section{La clasificación tipológica según Lowie y Murdock}

17. 'Siglo xvi' no se clasificó, ya que en su terminología no se encontraron los lexemas correspondientes a los tíos, tanto paterno como materno, necesarios para la tipología de Lowie, así como tampoco los términos de la hermana y las primas, paralela y cruzada, para la propuesta de Murdock. En el caso de 'Chicomucelteco', tampoco se clasificó según los criterios de Lowie, por faltar el término del tío materno. De acuerdo 
con este último autor, 'Tapia Zenteno', 'Siloxúchil', 'Tamaletón' y 'Loma Larga' tipifican el sistema como colateral bifurcado, pero en 'San Francisco' es lineal, lo que significa una simplificación del sistema.

Aplicando la clasificación de Murdock, es esquimal en 'Chicomucelteco', 'Loma Larga' y 'Tapia Zenteno', pero en éste sólo cuando se trata de ego femenino; por último, es hawaiano en 'Tamaletón', 'Siloxúchil', 'San Francisco' y únicamente para ego masculino en 'Tapia Zenteno'. Con respecto a la propuesta de Murdock, se observan dos tendencias en 'Tapia Zenteno': una más conservadora con el tipo hawaiano y otra más evolucionista con el tipo esquimal. 'Tamaletón', 'Siloxúchil' y 'San Francisco' siguen la primera tendencia, mientras que 'Chicomucelteco' y 'Loma Larga' la segunda. Lo anterior permite considerar como más conservador a 'Tamaletón' y en un plano de transición a 'Loma Larga' y a 'San Francisco'. Lo anterior se ilustra en el cuadro 3.

Cuadro 3. Clasificación tipológica de los sistemas de parentesco de la rama huastecana

\begin{tabular}{|c|c|c|c|c|c|c|c|}
\hline AUTOR & $\begin{array}{l}\text { JUAN DE } \\
\text { LA CRUZ } \\
\text { S. XVI }\end{array}$ & $\begin{array}{l}\text { T.ZENTENO } \\
\text { S. XVIII }\end{array}$ & $\begin{array}{l}\text { CHICOMUCELTECO } \\
\text { S. } X I X-X X\end{array}$ & $\begin{array}{l}\text { SILOXUCHIL } \\
\text { S. } \mathrm{xx}\end{array}$ & $\begin{array}{l}\text { TAMALETON } \\
\text { S. } \mathrm{xX}\end{array}$ & $\begin{array}{l}\text { LOMA } \\
\text { LARGA } \\
\text { S. } x x\end{array}$ & $\begin{array}{l}\text { SAN } \\
\text { FRANCISCO } \\
\text { S. } \mathrm{XX}\end{array}$ \\
\hline LOWIE & & $\begin{array}{l}\text { colateral } \\
\text { bifurcado }\end{array}$ & $x^{2}$ & $\begin{array}{l}\text { colateral } \\
\text { bifurcado }\end{array}$ & $\begin{array}{l}\text { colateral } \\
\text { bifurcado }\end{array}$ & $\begin{array}{l}\text { colateral } \\
\text { bifurcado }\end{array}$ & lineal \\
\hline MURDOCK & & $\begin{array}{l}\text { ego of: hawaiano } \\
\text { ego of: esquimal }\end{array}$ & esquimal & hawaiano & hawaiano & esquimal & hawaiano \\
\hline
\end{tabular}

$X$. En los cuadros 4 y 5 se muestran las equivalencias entre las grafías de los fonemas del huasteco y las empleadas por los otros autores en la terminología de parentesco de los siete sistemas de la rama huastecana. Según la propuesta fonológica de Ochoa (1984: 11-37) para la variante veracruzana, y la de Edmonson (1988:30-31) para la potosina (nota 1), se tiene lo siguiente (sobre el material del chicomucelteco, se indican los autores que emplean esa grafía y cuando no se hace corresponden a Sapper).

\section{Conclusiones}

Si bien en el inciso IX se comentaron detalladamente los aspectos relevantes del presente estudio, en estas conclusiones se hace una síntesis de ellos:

Todos los sistemas de parentesco expresan cinco categorías de Kroeber (1952: 175-181) (generación, consanguíneo/afín, lineal/ colateral, sexo del hablante y sexo del pariente). 'Tapia Zenteno' y 'Chicomucelteco' son los únicos que incluyen la categoría edad relativa dentro de una misma generación. 'Chicomucelteco' y 'San Francisco' no proporcionaron la categoría sexo del pariente que da la relación de parentesco y en ningún sistema se documentó la categoría condición de vida de la persona que relaciona. 
Cuadro 4. Correspondencias entre las grafías empleadas en el texto y las de los fonemas del huaxteco

\begin{tabular}{|c|c|c|c|c|c|}
\hline $\begin{array}{c}\text { XVI } \\
\text { Cruz }\end{array}$ & Tapia Zenteno & $\begin{array}{c}\text { XIX-xx } \\
\text { Sapper/Termer }\end{array}$ & $\begin{array}{c}\mathrm{xx} \\
\text { Guiteras }\end{array}$ & $\begin{array}{c}\mathrm{xX} \\
\text { Anzaldo }\end{array}$ & Fonemas \\
\hline $\mathrm{i}$ & $\mathrm{i}$ & $\mathrm{i}(\mathrm{S} / \mathrm{T})$ & $\mathrm{i}$ & $\mathrm{i}$ & $\mathrm{i}$ \\
\hline $\mathrm{i}$ & $\mathrm{i}$ & & $\mathrm{i}$ & $\mathrm{ii}$ & $\mathrm{i}:$ \\
\hline $\mathrm{u} / \mathrm{v}$ & $\mathrm{u}$ & $\mathrm{u}(\mathrm{S} / \mathrm{T}) / \mathrm{u}(\mathrm{T})$ & $\mathrm{u}$ & $\mathrm{u}$ & $\mathrm{u}$ \\
\hline & $\mathrm{u}$ & & $\mathrm{u}$ & $\mathrm{uu}$ & $\mathrm{u}$ \\
\hline & $\mathrm{e}$ & $\mathrm{e} / \mathrm{e}$ & $\mathrm{e} / \mathrm{e}$ & $\mathrm{e}$ & $\mathrm{e}$ \\
\hline $\mathrm{a} / \mathrm{o}$ & $\mathrm{a} / \mathrm{o}$ & $\mathrm{o}$ & $\mathrm{o}$ & $\mathrm{o}$ & $\mathrm{o}$ \\
\hline $\mathrm{o}$ & $\mathrm{o}$ & $\mathrm{a}$ & $\mathrm{o}$ & $\mathrm{oo}$ & $\mathrm{o}$ \\
\hline $\mathrm{a}$ & $\mathrm{a}$ & $\mathrm{a}(\mathrm{S} / \mathrm{T})$ & $\mathrm{a}$ & $\mathrm{a}$ & $\mathrm{a}$ \\
\hline $\mathrm{a}$ & $\mathrm{a}$ & $\mathrm{a}$ & $\mathrm{a} / \mathrm{aa}$ & $\mathrm{aa}$ & $\mathrm{a}$ \\
\hline $\mathrm{u} / \mathrm{v} / \mathrm{hu} / \mathrm{gu}$ & $\mathrm{u}$ & & & $\mathrm{w}$ & $\mathrm{w}$ \\
\hline $\mathrm{y} / \mathrm{i}$ & $\mathrm{y}$ & $(\mathrm{S} / \mathrm{T}) \mathrm{y} / \mathrm{i}$ & $\mathrm{y}$ & $\mathrm{y}$ & $\mathrm{y}$ \\
\hline
\end{tabular}

Cuadro 5. Correspondencias entre las grafías empleadas en el texto y las de los fonemas del huaxteco

\begin{tabular}{|c|c|c|c|c|c|}
\hline $\begin{array}{c}\mathrm{xVI} \\
\text { Cruz }\end{array}$ & $\begin{array}{c}\text { XVIII } \\
\text { Tapia Zanteno }\end{array}$ & $\begin{array}{c}\text { XIX-xx } \\
\text { Sapper/Termer }\end{array}$ & $\begin{array}{c}\mathrm{XX} \\
\text { Guiteras }\end{array}$ & $\begin{array}{c}\mathrm{xx} \\
\text { Anzaldo }\end{array}$ & Fonemas \\
\hline $\mathrm{p}$ & $\mathrm{p}$ & $\mathrm{p}$ & $\mathrm{p}$ & $\mathrm{p}$ & $\mathrm{p}$ \\
\hline$t$ & $t$ & $t(S / T)$ & $\mathrm{t}$ & $t$ & $\mathrm{t}$ \\
\hline $\mathrm{t}$ & $\mathrm{t}$ & $t$ & & $t^{\prime}$ & $t^{\prime}$ \\
\hline \multirow[t]{4}{*}{$\mathrm{c} / \mathrm{qu}$} & c & $(\mathrm{S} / \mathrm{T}) \mathrm{c} / \mathrm{k}(\mathrm{S} / \mathrm{T})$ & k & k & k \\
\hline & c & 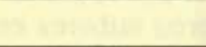 & k & $k^{\prime}$ & $k^{\prime}$ \\
\hline & 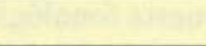 & 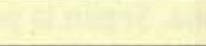 & 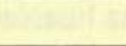 & $k^{w}$ & $k^{w}$ \\
\hline & 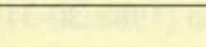 & $=$ & & , & $?$ \\
\hline \multirow[t]{5}{*}{$\mathrm{b} / \mathrm{v}$} & $\mathrm{b} / \mathrm{p}$ & ú* & b & b & b \\
\hline & $\mathrm{tz}$ & & & ts & $c$ \\
\hline & $\mathrm{tz}$ & & & ts' & $c^{\prime}$ \\
\hline & ch & $\operatorname{ch}(\mathrm{S} / \mathrm{T})$ & ch & ch & $\check{c}$ \\
\hline & & & ch' & ch' & č' \\
\hline$\zeta / z$ & $\mathrm{z}$ & y & $\mathrm{z}$ & $\mathrm{dh}$ & $\theta$ \\
\hline \multirow[t]{2}{*}{$s / x$} & $\mathrm{x}$ & $\mathrm{x}$ & $\mathrm{x}$ & $x$ & $\check{s}$ \\
\hline & $\mathrm{x} / \mathrm{h}$ & j & j & $\mathrm{j}$ & $\mathrm{h}$ \\
\hline \multirow[t]{2}{*}{$\mathrm{m} / \mathrm{TILDE}$} & $\mathrm{m}$ & $\mathrm{m}$ & $\mathrm{m}$ & $\mathrm{m}$ & $\mathrm{m}$ \\
\hline & $\mathrm{n}$ & $n(S / T)$ & $\mathrm{n}$ & $\mathrm{n}$ & $\mathrm{n}$ \\
\hline ИI & $1 / 1$ & $\mathrm{n} / /^{* *}$ & 1 & 1 & 1 \\
\hline
\end{tabular}

* Véase nota 18

* Véase nota 19 


\section{Cuadro 6. Comparacion de los términos de parentesco de las lenguas de la rama huastecana}

\begin{tabular}{|c|c|c|c|c|c|c|c|}
\hline Pariente & Siglo xvi & $\begin{array}{c}\text { Tapia } \\
\text { Zenteno }\end{array}$ & $\begin{array}{l}\text { Chicomu- } \\
\text { celteco }\end{array}$ & Siloxúchil & Tamaletón & $\begin{array}{l}\text { Loma } \\
\text { Larga }\end{array}$ & $\begin{array}{c}\text { San } \\
\text { Francisco }\end{array}$ \\
\hline $\begin{array}{l}\mathrm{PP}(\mathrm{M}) \mathrm{P}(\mathrm{M}) \mathrm{P}(\mathrm{M}) \\
\text { "tatarabuelo" }\end{array}$ & & & & oxil maam & oxil pulek* & oxil* & \\
\hline $\begin{array}{l}\text { MP(M)P(M)P(M) } \\
\text { "tatarabuela" }\end{array}$ & & & & oxil aach & oxil pulek & oxil* & \\
\hline $\begin{array}{l}\mathrm{PP}(\mathrm{M}) \mathrm{P}(\mathrm{M}) \\
\text { "bisabuelo" }\end{array}$ & & tzabilmam & & $\begin{array}{l}\text { chab pulék } \\
\text { taata }\end{array}$ & tsabil maam & $\begin{array}{l}\text { chaabil pulek } \\
\text { taata }\end{array}$ & chaabil* \\
\hline $\begin{array}{l}\text { MP(M)P(M) } \\
\text { "bisabuela" }\end{array}$ & & tzabilach & & $\begin{array}{l}\text { chab pulék } \\
\text { naana }\end{array}$ & tsabil aach & $\begin{array}{l}\text { chaabil pulek } \\
\text { naana }\end{array}$ & chaabil* \\
\hline $\begin{array}{l}\text { PP(M) } \\
\text { "abuelo" }\end{array}$ & & mam & mam & pulék taata & maam & pulek taata & $\begin{array}{l}\text { maam } \\
\text { pulik paap }\end{array}$ \\
\hline $\begin{array}{l}\text { MP(M) } \\
\text { "abuela" }\end{array}$ & & ach & xapin & pulék naana & aach & pulek naana & $\begin{array}{l}\text { aach } \\
\text { pulik ma }\end{array}$ \\
\hline $\begin{array}{l}\text { P } \\
\text { "padre" }\end{array}$ & $\begin{array}{l}\delta \text { paylom } \\
\Varangle \text { pap }\end{array}$ & $\begin{array}{l}\delta \text { paylom } \\
\Varangle \text { pap }\end{array}$ & ajtic & taata & $\begin{array}{l}\text { taata } \\
\text { pay'loom }\end{array}$ & taata & $\begin{array}{l}\text { paap } \\
\text { paapa }\end{array}$ \\
\hline $\begin{array}{l}\text { M } \\
\text { "madre" }\end{array}$ & $\mathrm{mim}$ & $\operatorname{mim}$ & epé & naana & naana $\sim$ miim & naana & $\begin{array}{l}\text { ma } \\
\text { maama }\end{array}$ \\
\hline $\begin{array}{l}\text { HoP } \\
\text { "tío paterno" }\end{array}$ & & & & paylóm & $\begin{array}{l}\text { ts'uts' } \\
\text { pay'loom }\end{array}$ & $\begin{array}{l}\text { ot pay'loom* } \\
\text { \& paap* }\end{array}$ & ichaan ${ }^{\bullet}$ \\
\hline $\begin{array}{l}\text { Ho }>\text { P } \\
\text { "tío paterno } \\
\text { mayor" }\end{array}$ & & $\begin{array}{l}\delta \text { yelam paylom } \\
\text { } \text { yelam pap }\end{array}$ & & & & & \\
\hline $\begin{array}{l}\text { Ho }<\mathrm{P} \\
\text { "tío paterno } \\
\text { menor" }\end{array}$ & & $\begin{array}{l}\delta \text { tzutz paylom } \\
q \text { tzutz pap }\end{array}$ & & & & & \\
\hline $\begin{array}{l}\text { HoM } \\
\text { "tío materno" }\end{array}$ & & itzan & icháu & ichán & itsaan & ichaan & ichaan* \\
\hline $\begin{array}{l}\text { HaP } \\
\text { "tía paterna" }\end{array}$ & & tzanub* & & chanúb & tsanuub & chanuub & chanuub* \\
\hline $\begin{array}{l}\text { HaM } \\
\text { "tía materna" }\end{array}$ & & tzanub* & & $\operatorname{mim}$ & ts'uts' miim & miim* & chanuub* \\
\hline $\begin{array}{l}\text { EoM } \\
\text { "padrastro" }\end{array}$ & & $\begin{array}{l}\text { ó paylomle } \\
\text { \& paple }\end{array}$ & & chab taata & tatle' & $\begin{array}{l}\text { chab taata } \\
\delta \text { pay'loom } \\
\text { taata } \\
\text { क paap taata }\end{array}$ & paple' \\
\hline
\end{tabular}

El asterico (") indica que tiene más de un referente.

Estudios de Cultura Maya. Vol. XXIII, 2003

Instituto de Investigaciones Filológicas/

Centro de Estudios Mayas, UNAM

ISSN 0185-2574 


\section{Cuadro 7. Comparación de los términos de parentesco de las lenguas de la rama huastecana}

\begin{tabular}{|c|c|c|c|c|c|c|c|}
\hline Pariente & $\begin{array}{l}\text { Siglo } \\
x \mathrm{x} 1\end{array}$ & $\begin{array}{c}\text { Tapia } \\
\text { Zenteno }\end{array}$ & $\begin{array}{l}\text { Chicomu- } \\
\text { celteco }\end{array}$ & Siloxúchil & Tamaletón & $\begin{array}{l}\text { Loma } \\
\text { Larga }\end{array}$ & $\begin{array}{c}\text { San } \\
\text { Francisco }\end{array}$ \\
\hline $\begin{array}{l}\text { EaP } \\
\text { "madrastra" }\end{array}$ & & mimle & & chab naana & mimle' & $\begin{array}{l}\text { chab naana } \\
\text { miim naana }\end{array}$ & mimle' \\
\hline $\begin{array}{l}\text { PEo(a) } \\
\text { "suegro" }\end{array}$ & & $\begin{array}{l}\delta \text { iyam } \\
q \text { alib }\end{array}$ & iyam & $\begin{array}{l}o ̛ \text { taata-iyám } \\
\text { q taata-alíb }\end{array}$ & $\begin{array}{l}\delta \text { iyaam* } \\
q \text { inik al'iib }\end{array}$ & $\begin{array}{l}\text { ó pay'loom* } \\
\text { \& paap* }\end{array}$ & $\begin{array}{l}o \text { iyaam* } \\
\text { o inik al'iib }\end{array}$ \\
\hline $\begin{array}{l}\text { MEo(a) } \\
\text { "suegra" }\end{array}$ & & $\begin{array}{l}\delta \text { uxum iyam } \\
\text { i uxum alib }\end{array}$ & iyam & $\begin{array}{l}\text { ơ naana-iyám } \\
\text { § naana-alíb }\end{array}$ & $\begin{array}{l}\delta \text { uxum iyaam } \\
q \text { uxum al'iib }\end{array}$ & miim* & $\begin{array}{l}\delta \text { uxum iyaam } \\
\text { o uxum al'iib }\end{array}$ \\
\hline $\begin{array}{l}\text { Ho } \\
\text { "hermano" }\end{array}$ & quiçab & $\delta$ xibam & uktak & $\begin{array}{l}\text { kizáb* } \\
\text { ơ atál* } \\
\text { † chu'ch"* }\end{array}$ & $\begin{array}{l}\delta \text { ataatal* } \\
\text { \& inik ebchal* }\end{array}$ & $\begin{array}{l}\delta \text { ataal* } \\
\text { o kidhaab* }\end{array}$ & ebal* \\
\hline $\begin{array}{l}\text { Ho> } \\
\text { "hermano mayor" }\end{array}$ & & $\delta$ at mim* & cocú & & $\delta$ at miim** & & \\
\hline $\begin{array}{l}\mathrm{Ho}< \\
\text { "hermano menor" }\end{array}$ & & $\delta$ atatal ${ }^{*}$ & acaú & & & & \\
\hline $\begin{array}{l}\text { Ha } \\
\text { "hermana" }\end{array}$ & & ơ ixam* & nunú & $\begin{array}{l}\text { kizáb* } \\
\text { ơ ixám* } \\
\text { † bayil* }\end{array}$ & ebchal* & kidhaab* & ebal* \\
\hline $\begin{array}{l}\mathrm{Ha}> \\
\text { "hermana mayor" }\end{array}$ & & ㅇ bayil & & & & & \\
\hline $\begin{array}{l}\mathrm{Ha}< \\
\text { "hermana menor" }\end{array}$ & & q acab & & & & & \\
\hline $\begin{array}{l}\text { hoHo(a)P(M) } \\
\text { "primo" }\end{array}$ & & $\begin{array}{l}\delta^{*} \text { at mim } \\
\delta^{*} \text { atatal } \\
\& \text { epchial* }\end{array}$ & uicha* & $\begin{array}{l}\text { ơ atál* } \\
\text { ๆ chu'ch** }\end{array}$ & $\begin{array}{l}\delta \text { ataatal } \\
\text { \& inik ebchal* }\end{array}$ & $\begin{array}{l}\delta \text { ataal* } \\
\text { \& ch'uuch' }\end{array}$ & ebal* \\
\hline $\begin{array}{l}\text { haHo(a)P(M) } \\
\text { "prima" }\end{array}$ & & $\begin{array}{l}\delta^{*} \text { ixam* } \\
\text { \& epchial* }\end{array}$ & uicha* & $\begin{array}{l}\text { ơ ixám* } \\
\text { † bayil* }\end{array}$ & ebchal* & $\begin{array}{l}\delta \text { ixaam* } \\
\text { o bayil* }\end{array}$ & ebal* \\
\hline $\begin{array}{l}\text { HoEo(a) } \\
\text { EoHa } \\
\text { "cuñado" }\end{array}$ & & $\begin{array}{l}o \text { bay } \\
\text { ot at mul* }\end{array}$ & úai & $\begin{array}{l}\delta^{*} \text { bayj } \\
\text { क at-mul* }\end{array}$ & $\begin{array}{l}\delta \text { baay } \\
q \text { xibaam }\end{array}$ & $\begin{array}{l}\delta \text { baay } \\
q \text { at muul }\end{array}$ & $\begin{array}{l}\text { baay' } \\
\delta \text { ch'uuch' }\end{array}$ \\
\hline $\begin{array}{l}\text { HaEo(a) } \\
\text { EaHo } \\
\text { "cuñada" }\end{array}$ & & $\begin{array}{l}\delta \text { at mul } \\
\& \text { xahuan }\end{array}$ & atmún & $\begin{array}{l}\delta^{*} \text { at-mul* } \\
\text { † jauán }\end{array}$ & $\begin{array}{l}\delta \text { ixaam } \\
q \text { jawan }\end{array}$ & $\begin{array}{l}\delta \text { at muul* } \\
\& \text { jawan }\end{array}$ & $\begin{array}{l}\text { ơ ixaam* } \\
\text { क jawan * }\end{array}$ \\
\hline
\end{tabular}

•*Este término designa al medio hermano materno 


\section{Cuadro 8. Comparación de los términos de parentesco de las lenguas de la rama huastecana}

\begin{tabular}{|c|c|c|c|c|c|c|c|}
\hline Pariente & Siglo xvı & $\begin{array}{c}\text { Tapia } \\
\text { Zenteno }\end{array}$ & $\begin{array}{l}\text { Chicomu- } \\
\text { celteco }\end{array}$ & Siloxúchil & Tamaletón & $\begin{array}{l}\text { Loma } \\
\text { Larga }\end{array}$ & $\begin{array}{c}\text { San } \\
\text { Francisco }\end{array}$ \\
\hline $\begin{array}{l}\text { Eo } \\
\text { "esposo" }\end{array}$ & $\begin{array}{l}\text { pizob* } \\
\wp \text { illau }\end{array}$ & $\begin{array}{l}\text { tomol } \sim \text { pizob* } \\
\uparrow \text { illauh }\end{array}$ & $q$ tetlom & tomkil* & $\begin{array}{l}\text { १ ilow } \\
\text { tomkiil* }\end{array}$ & $\begin{array}{l}\text { tomkiil० } \\
\text { tomtal }\end{array}$ & $\begin{array}{l}\text { tomkiil" } \\
\text { \& yejchelel }\end{array}$ \\
\hline $\begin{array}{l}\text { Ea } \\
\text { "esposa" }\end{array}$ & $\begin{array}{l}\text { pizob" } \\
\delta \text { vxmal }\end{array}$ & $\begin{array}{l}\text { tomol pizob* } \\
\delta^{*} \text { ixal }\end{array}$ & ¿uxmal & tomkil" & $\begin{array}{l}\delta \text { iixal } \\
\text { tomkiil• }\end{array}$ & $\begin{array}{l}\text { tomkiil• } \\
\text { tomtal }\end{array}$ & $\begin{array}{l}8 \text { ixal } \\
\text { tomkiil* }\end{array}$ \\
\hline $\begin{array}{l}\text { PEo(a)ho(a) } \\
\text { "consuegro" }\end{array}$ & & atsii & & at-chij & kumpaale atsii & kumpaale & kumpaale \\
\hline $\begin{array}{l}\text { MEo(a)ho(a) } \\
\text { "consuegra" }\end{array}$ & & uxum atzii & & & kumaale atsii & kumaale & kumaale \\
\hline $\begin{array}{l}\text { hoEo(a)P(M) } \\
\text { "hermanastro" }\end{array}$ & . & 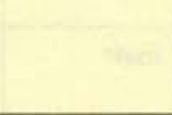 & & & $\begin{array}{l}\text { ot ataatle' }^{\prime} \\
q^{\prime} \text { inik ebchale' }\end{array}$ & $\begin{array}{l}\text { o chab } \\
\text { kidhaab* } \\
\delta \text { chab ataal }\end{array}$ & $\begin{array}{l}\text { pulik ebal } \\
\text { ch'ejel ebal' }\end{array}$ \\
\hline $\begin{array}{l}\text { haEo(a)P(M) } \\
\text { "hermanastra" }\end{array}$ & & & & & ebchale' & chab kidhaab* & $\begin{array}{l}\text { pulik ebal } \\
\text { ch'ejel ebal* }\end{array}$ \\
\hline $\begin{array}{l}\text { EoHaEo(a) } \\
\text { "concuño" }\end{array}$ & & atie atiyam & & $\delta^{8}$ at-iyám ${ }^{26}$ & $\begin{array}{l}\delta \text { at iya } ~ \\
\text { at muul } \\
q \text { xibaam* }\end{array}$ & $\begin{array}{l}\delta \text { ataal } \sim \\
\text { at iya } \\
q \text { at mul* }\end{array}$ & $\begin{array}{l}\text { o ch'uuch"* } \\
\text { at iya } \\
\text { क baay" }\end{array}$ \\
\hline $\begin{array}{l}\text { EaHoEo(a) } \\
\text { "concuña" }\end{array}$ & & 8 at alib $^{27}$ & & $\uparrow$ at-alíb ${ }^{27}$ & $\begin{array}{l}\text { o ixaam* }^{*} \\
\text { o bayil }\end{array}$ & $\begin{array}{l}\delta \text { ixaam* } \\
\text { o bayil } \sim \\
\text { at al'iib }\end{array}$ & $\begin{array}{l}\delta^{*} \text { ixaam* } \\
\text { o jawan }\end{array}$ \\
\hline $\begin{array}{l}\text { ho } \\
\text { "hijo" }\end{array}$ & $\begin{array}{l}\text { of atic } \\
\text { o tam }\end{array}$ & $\begin{array}{l}\delta \text { atic } \\
\text { o taam }\end{array}$ & q tam* & chakámel• & $\begin{array}{l}\text { kwitoolil } \\
\text { atik* } \\
\text { tsakaamil* }\end{array}$ & $\begin{array}{l}\text { kwitoolil } \sim \\
\text { chakaamil* }\end{array}$ & $\begin{array}{l}\text { kwitoolil } \\
\text { chikaamil" }\end{array}$ \\
\hline $\begin{array}{l}\text { ho> } \\
\text { "hijo mayor" }\end{array}$ & & $\begin{array}{l}\text { of cochim atic } \\
\text { o cochim tam }\end{array}$ & & & & & \\
\hline $\begin{array}{l}\text { ho< } \\
\text { "hijo menor" }\end{array}$ & & $\begin{array}{l}\text { o tzutzu atic } \\
\text { o tzutz tam }\end{array}$ & & & & & \\
\hline $\begin{array}{l}\text { ha } \\
\text { "hija" }\end{array}$ & & & $\operatorname{tam}^{*}$ & chakámel• & $\begin{array}{l}\text { ts'ik'aachil } \\
\text { atik* } \\
\text { tsakaamil* }\end{array}$ & $\begin{array}{l}\text { tsidhaanil } \\
\text { chakaamil* }\end{array}$ & $\begin{array}{l}\text { chidhaanil } \\
\text { chikaamil }\end{array}$ \\
\hline
\end{tabular}

\section{${ }^{26}$ Solamente EoHaEa \\ ${ }^{27}$ Solamente EaHoEo}


Cuadro 9. Comparación de los términos

de parentesco de las lenguas de la rama huastecana

\begin{tabular}{|c|c|c|c|c|c|c|c|}
\hline Pariente & $\begin{array}{l}\text { Siglo } \\
x \mathrm{x}\end{array}$ & $\begin{array}{c}\text { Tapia } \\
\text { Zenteno }\end{array}$ & $\begin{array}{l}\text { Chicomu- } \\
\text { celteco }\end{array}$ & Siloxúchil & Tamaletón & $\begin{array}{l}\text { Loma } \\
\text { Larga }\end{array}$ & $\begin{array}{c}\text { San } \\
\text { Francisco }\end{array}$ \\
\hline $\begin{array}{l}\text { hoHo } \\
\text { "sobrino, hijo } \\
\text { del hermano" }\end{array}$ & & \% iyac & & $\begin{array}{l}\text { ơ chakámel* } \\
\text { † ichák* }\end{array}$ & itsak"* & ichak" & ichak" \\
\hline $\begin{array}{l}\text { hoHa } \\
\text { "sobrino, hijo } \\
\text { de la hermana" }\end{array}$ & & $\delta$ itzac & & $\begin{array}{l}\text { ơ ichák* } \\
\text { \& chakámel• }\end{array}$ & itsak"* & ichak"* & ichak"* \\
\hline $\begin{array}{l}\text { haHo } \\
\text { "sobrina, hija } \\
\text { del hermano" }\end{array}$ & & & & $\begin{array}{l}\text { ơ chakámel } \\
\text { @ ichák• }\end{array}$ & itsak" & ichak" & ichak"* \\
\hline $\begin{array}{l}\text { haHa } \\
\text { sobrina, hija } \\
\text { de la hermana" }\end{array}$ & & & & $\begin{array}{l}\text { ơ ichák* } \\
\text { o chakámel* }\end{array}$ & itsak* & ichak"* & ichak"* \\
\hline $\begin{array}{l}\text { Eoha } \\
\text { "yerno" }\end{array}$ & & iyam & iyam & iyám & iyaam* & iyaam & iyaam* \\
\hline $\begin{array}{l}\text { Eaho } \\
\text { "nuera" }\end{array}$ & & alib & & alíb & al'iib & al'iib & al'iib \\
\hline $\begin{array}{l}\text { hoEo(a) } \\
\text { "hijastro" }\end{array}$ & & & & chab chakámel* & $k^{w}$ itotle' & chab $k^{w}$ itoolil & t'amle" \\
\hline $\begin{array}{l}\text { haEo(a) } \\
\text { "hijastra" }\end{array}$ & & 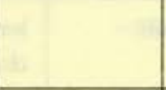 & 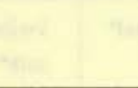 & chab chakámel & ts'ik'aachle' & chab tsidhaanil & t'amle'* \\
\hline $\begin{array}{l}\text { ho(a)ho(a) } \\
\text { "nieto/a" }\end{array}$ & & $\begin{array}{l}8 \text { momob } \\
\$ \text { iyib }\end{array}$ & iú & momob & momob & momob & momob \\
\hline $\begin{array}{l}\text { ho(a)ho(a)ho(a) } \\
\text { "bisnieto/a" }\end{array}$ & & tzabilvmomob & & & tsabil momob & chaabil momob & chaabil \\
\hline $\begin{array}{l}\text { ho(a)ho(a)ho(a)ho(a) } \\
\text { "tataranieto/a" }\end{array}$ & & & & & oxil momob & oxil momob & oxil \\
\hline
\end{tabular}


'Tamaletón', 'Loma Larga' y 'San Francisco' registran más generaciones (9) que los demás sistemas. 'Tapia Zenteno' expresa en un $71 \%$ la categoría sexo del hablante, lo cual informa sobre la profunda división entre el habla de los hombres y las mujeres.

Es frecuente que con el mismo término se designen los hermanos y los primos, situación que se acentúa en 'San Francisco'.

La estructura y la terminología de los cuñados es la misma en 'Tapia Zenteno', 'Siloxúchil' y 'Loma Larga'. 'San Francisco' realiza cambios en los términos de esos parientes.

'Siloxúchil' diferencia los sobrinos paralelos de los cruzados y 'Tapia Zenteno' solamente proporciona los términos de los sobrinos cruzados masculinos.

En 'Loma Larga' se denominan parientes afines con términos consanguíneos $(9 \mathrm{~F}, 12 \mathrm{~F}$, $26 \mathrm{~F}, 27 \mathrm{~F}, 38 \mathrm{~F}, 39 \mathrm{~F}$ ) lo que sugiere la presencia del matrimonio entre primos cruzados.

El estudio comparativo sobre la terminología de los cuñados en 11 lenguas mayas (cuadro 2) mostró que el término jawan 'cuñada de ego femenino', pertenece a los sistemas siguientes: maya-yucateco del siglo xvı, maya mopán, tzeltal, tzotzil y ch'ol. Pero además, el mismo término con igual referencia es parte del sistema huasteco, pues ya aparece desde el siglo xvill. Con respecto a 'Chicomucelteco', sólo proporcionó el término atmún 'cuñada', sin especificar el sexo del hablante. Los sistemas 'Tapia Zenteno', 'Siloxúchil' y 'Loma Larga', que, como antes se dijo, tienen idéntica terminología para los cuñados, incluyen ese último término para nombrar al pariente de sexo opuesto de ego masculino y para el femenino emplean jawan. Es muy probable que una de las personas que entrevistó Sapper fuera del sexo masculino ya que también para el cuñado obtuvo un solo término, úai, que precisamente en los sistemas citados corresponde a ego masculino. En caso de que esa fuera la situación, se podría afirmar que el término jawan es parte del sistema terminológico de la rama huastecana.

El número de cognadas parciales es considerable (54\%). 'Siloxúchil' (85\%) y 'Loma Larga' $(80 \%)$, ambos sistemas del municipio de Tantoyuca, tienen alto porcentaje de cognadas. No así 'San Francisco' (73\%), también de Veracruz pero del municipio de Chontla. Entre 'Tapia Zenteno' y 'Tamaletón' es alto el porcentaje de términos cognados (62\%), 24 son cognadas de forma y significado y siete solamente de forma.

De la comparación terminológica resultaron dos cognadas de forma y significado en seis sistemas, excluido 'Siglo xv', una que nombra al tío materno y la otra al cuñado de ego masculino. Queda por investigar si la estabilidad del término del tío materno está relacionada con el avunculado.

De acuerdo con la propuesta tipológica de Lowie los sistemas con los datos pertinentes resultaron del tipo colateral bifurcado. La tipología de Murdock osciló entre el tipo hawaiano y el esquimal.

Finalmente, el sistema más dinámico es el de 'Loma Larga' y el más conservador el de 'Tamaletón'.

\section{BIBLIOGRAFÍA}

Anzaldo Figueroa, Rosa Elena y Fidencio Briceño Chel

1998 "Comparación de los sistemas de parentesco huaxteco y maya", en J. Ruvalcaba Mercado (ed.), Nuevos aportes al conocimiento de la Huaxteca, pp. 203-219, México, CIESAS. 
Blair, Robert W., John S. Robertson et al.

1981 Diccionario español-cakchiquel-inglés, Nueva York, Garland.

Borodatova, Anna e Irene Kozhanovskaya

1999 "El protosistema de parentesco maya: tentativa de reconstrucción", en Estudios de Cultura Maya, vol. XX, pp. 332-364, México, UNAM-IIFI-Centro de Estudios Mayas.

Campbell, Lyle y Marianne Mithun (eds.)

1979 The Languages of Native America: Historical and Comparative Assesment, Austin, University of Texas Press.

De la Cruz, fray Juan

1571 Doctrina christiana en la lengua guasteca con la lengua castellana, México, Imp. Pedro Ocharte.

Esponda, Víctor Manuel

1996 "Los sistemas omaha entre los mayas de Los Altos de Chiapas y una consideración sobre los ch'oles", en J. Jáuregui, Ma. E. Olavarría y V. M. Franco Pellotier (eds.), Cultura y comunicación. Edmund Leach: in memoriam, pp.105-120, México, CIESAS/UAM.

Edmonson, Barbara

1988 "A Descriptive Grammar of Huastec (Potosino Dialect)", Michigan, Tulane University, tesis de doctorado.

Goodenough, Ward $\mathrm{H}$.

1956 "Componential Analysis and the Study of Meaning", en Language, núm. 32, pp. 195216.

Guiteras Holmes, Calixta

1948 "Sistema de parentesco huaxteco", en Acta Americana, núms. 3-4, vol. VI, pp. $152-$ 172 México,

1966 "Cambio de un sistema omaha a un sistema bilateral entre los tzotziles de Chiapas", en J. Jáuregui y J. del Val (eds.), Los estudios de parentesco en México, pp. 155-178, México, Sociedad Mexicana de Antropología.

Hurley vda. De Delgaty, Alfa y Agustín Ruiz Sánchez

1978 Diccionario tzotzil de San Andrés con variaciones dialectales, México, ILV/SEP.

INEGI

1990 XI Censo general de población y vivienda, 1990, México.

Jeffers, Robert J. y llse Lehiste

1982 Principles and Methods for Historical Linguistics, Cambridge, Mas.-Londres, The MIT Press.

Kaufman, Terrence

1972 El proto-tzeltal-tzotzil. Fonología comparada y diccionario reconstruido, México, UNAMIIFI-Centro de Estudios Mayas.

Kroeber, A. L.

1944 "The Historical Position of Chicomuceltec in Mayan", en IJAL, núm. 4, vol. 10, pp. 159-160, Baltimore.

1952 "Classificatory Systems of Relationship", en The Nature of Culture, pp. 175-181,Chicago-London, The University of Chicago Press.

Larsen, Raymond

1955 Vocabulario huaxteco del estado de San Luis Potosí, México, ILV/SEP. 
Lévi-Strauss, Claude

1969 Antropología estructural, Buenos Aires, Universitaria de Buenos Aires.

Lounsbury, F. G.

1964a "The Structural Analysis of Kinship Semantics", en H. G. Lunt (ed.), Proceedings of the IXth International Congress of Linguistics, pp.193-211, La Haya, Mouton.

1964b "A Formal Account of the Crow-Omaha Type Kinship Terminologies", en H. Goodenough (ed.), Explorations in Cultural Anthropology, pp. 212-255, McGraw-Hill, Nueva York.

Lowie, R. H.

1946 "Relationship Terms", en Encyclopaedia Britannica, vol. 19, Chicago, Helen Hermingway/Benton Publisher, $14^{2}$ ed., pp. 84-89.

Manrique Castañeda, Leonardo

1990 "La posición de la lengua huaxteca", en L. Ochoa (ed.), Huaxtecos y totonacos, pp. 206-224, México, Conaculta.

Medina Hernández, Andrés

1991 Tenejapa: familia y tradición de un pueblo tzeltal, México, Gob. del Edo. de ChiapasInstituto Chiapaneco de Cultura.

Miles, Suzanne W.

1983 Los pokomames del siglo XVI, Guatemala, Seminario de Integración Social Guatemalteca.

Montejano y Aguiñaga, Rafael

1960 "Notas de bibliografía lingüística huaxteca y el paradigma apologético del doctor Tapia Centeno", en Fichas de Bibliografia Potosina, núms. 3-4, vol. VI, pp. 50-84, México.

Murdock, G. P.

1965 Social Structure, Nueva York, The Free Press.

Ochoa Peralta, Ma. Ángela

1984 El idioma huaxteco de Xiloxúchil, Veracruz, México, INAH.

1995 "La doctrina christiana en la lengua guasteca de fray Juan de la Cruz; primicias de un análisis", en Amerindia, núms. 19-20, pp. 122-128.

Ruz, Mario Humberto

1982 Los legítimos hombres. Aproximación antropológica al grupo tojolabal, vol. II, México, UNAM, IIFI, Centro de Estudios Mayas.

1989 Las lenguas del Chiapas colonial. Manuscritos en la Biblioteca Nacional de París, vol.1, Lenguas mayances, México, UNAM, IIFI, Centro de Estudios Mayas.

Ruz, Mario Humberto (ed.)

1981 Los legítimos hombres. Aproximación antropológica al grupo tojolabal, vol. I, México, UNAM, IIFI, Centro de Estudios Mayas.

Sapper, Karl

1954 "Über einige Sprachen von Südchiapas", en Proceedings of the International Congress of Americanists, XVII sesión, parte 1, pp. 295-320. México.

Schumann Gálvez, Otto

1997 Introducción al maya mopán. Los itzaes desde la época prehispánica hasta la actualidad: estudio interdisciplinario de un grupo maya, México, UNAM-IIA.

Estudios de Cultura Maya. Vol. XXIII, 2003

Instituto de Investigaciones Filológicas/

Centro de Estudios Mayas, UNAM

ISSN 0185-2574 
Suárez, Jorge A.

1995 Las lenguas indígenas mesoamericanas, México, INI/CIESAS.

Swadesh, Morris

1960 "Interrelaciones de las lenguas mayences", en Anales del INAH, vol. 13, pp. 231-267, México, INAH.

Tapia Zenteno, Carlos de

1985 Paradigma apologético y noticia de la lengua huaxteca. Con vocabulario, catecismo y administración de sacramentos [reproducción facsimilar de la ed. de 1767], R. Acuña (ed.), México, UNAM.

Termer, Franz

1930 "Über die Mayasprache von Chicomucelo", Proceedings of the XXII International Congress of Americanists, pp. 926-936, Nueva York.

Wisdom, Charles

1961 Los chortis de Guatemala, Guatemala, Seminario de Integración Social Guatemalteca.

Zimmermann, Günter

1966 "El cotoque, la lengua mayence de Chicomucelo", en Traducciones Mesoamericanistas, vol. 1, pp. 27- 71, México, SMA. 